\title{
Transcriptomics, Targeted
} Metabolomics and Gene Expression of Blackberry Leaves and Fruits Indicate Flavonoid Metabolic Flux from Leaf to Red Fruit

\author{
Enrique Gutierrez ${ }^{1 *}$, Ana García-Villaraco ${ }^{1}$, José A. Lucas ${ }^{1}$, Ana Gradillas ${ }^{2}$, \\ F. Javier Gutierrez-Mañero ${ }^{1}$ and Beatriz Ramos-Solano ${ }^{1 *}$ \\ ${ }^{1}$ Plant Physiology, Pharmaceutical and Health Sciences Department, Faculty of Pharmacy, Universidad San Pablo-CEU \\ Universities, Madrid, Spain, ${ }^{2}$ Centre for Metabolomics and Bioanalysis, Pharmaceutical and Health Sciences Department, \\ Faculty of Pharmacy, Universidad San Pablo-CEU Universities, Madrid, Spain
}

OPEN ACCESS

Edited by:

Claudio Bonghi,

University of Padua, Italy

Reviewed by:

Lara Giongo,

Fondazione Edmund Mach, Italy

Ana Pina,

Centro de Investigación y Tecnología

Agroalimentaria de Aragón, Spain

*Correspondence:

Enrique Gutierrez

enrique.gutierrezalbanchez@ceu.es

Beatriz Ramos-Solano

bramso/@ceu.es

Specialty section:

This article was submitted to

Crop Science and Horticulture,

a section of the journal

Frontiers in Plant Science

Received: 31 January 2017

Accepted: 17 March 2017

Published: 06 April 2017

Citation:

Gutierrez E, García-Villaraco A, Lucas JA, Gradillas A,

Gutierrez-Mañero FJ and Ramos-Solano B (2017)

Transcriptomics, Targeted Metabolomics and Gene Expression of Blackberry Leaves and Fruits Indicate Flavonoid Metabolic Flux from Leaf to Red Fruit. Front. Plant Sci. 8:472. do: 10.3389/fpls.2017.00472
Blackberries (Rubus spp.) are among the high added value food products relevant for human health due to the increasing evidence of the beneficial effects of polyphenols, which are very abundant in these fruits. Interestingly, these compounds also play a role on plant physiology, being especially relevant their role in plant defense against biotic and abiotic stress. Hence, we hypothesize that since blackberry fruits have high amounts of flavonols and anthocyanins, leaves would also have high amounts of these compounds, and can be studied as a source of active molecules; furthermore, leaf synthesis would support their high contents in fruits. To explore this hypothesis, the present study reports a de novo transcriptome analysis on field grown blackberry leaves and fruits at the same time point, to establish the metabolic relationship of these compounds in both organs. Transcripts were aligned against Fragaria vesca genome, and genes were identified and annotated in different databases; tissue expression pattern showed 20,463 genes common to leaves and fruits, while 6,604 genes were significantly overexpressed only in fruits, while another 6,599 genes were significantly overexpressed in leaves, among which flavonol-anthocyanin transporter genes were present. Bioactives characterization indicated that total phenolics in leaves were three-fold, and flavonols were six-fold than in fruits, while concentration of anthocyanins was higher in fruits; HPLC-MS analysis indicated different composition in leaves and fruits, with cyanidin-3-glucoside as the only common compound identified. Next, RT-qPCR of the core genes in the flavonol anthocyanin pathway and regulatory MYB genes were carried out. Interestingly, genes in the flavonol-anthocyanin pathway and flavonol-transport families were overexpressed in leaves, consistent with the higher bioactive levels. On the other hand, transcription factors were overexpressed in fruits anticipating an active anthocyanin biosynthesis upon ripening. This suggests that, in addition to the biosynthesis taking place in the fruits during ripening, translocation of flavonols from leaves to fruits contributes to the high amounts of bioactives starting to accumulate in fruits.

Keywords: rubus, secondary metabolism, flavonoids, flavonols, anthocyanins, translocation, transcriptomics 


\section{INTRODUCTION}

Blackberry fruits are an important source of bioactive compounds, among which are flavonols, catechins, and anthocyanins (Garcia-Seco et al., 2013). These molecules have been reported to have beneficial effects to prevent metabolic syndrome at different levels such as cardiovascular disease and type II diabetes; hence, including blackberry on human diet is beneficial for health (Tavares et al., 2013; Feresin et al., 2016; Sarkar et al., 2016). On the other hand, they play a relevant role in plant defense against biotic and abiotic stress (Daayf et al., 2012). Catechins have also proved their improving endothelial function, hypertension, coronary heart disease, obesity, insulin resistance, as well as glucose and lipid metabolism (Kim, 2008).

Flavonols are a class of flavonoids, which have different roles in the green part of the plant, such as antioxidant, protecting the plant from reactive oxygen species (ROS), produced by abiotic stress like UV-B radiation, soil salinity, as a response to depletion of nitrogen in plants, as well as those ROS produced after biotic stress, as pathogen challenge, when they behave as phytoalexins (Wang and Lin, 2000; Gutiérrez-Mañero et al., 2015). They have also a growth regulating role, as they can act as auxin controllers, so they are able to control the individual plant organs and development of the whole plant (Agati et al., 2012; Falcone Ferreyra et al., 2012; Brunetti et al., 2013; Nakabayashi et al., 2014).

Despite the agricultural and biological importance of the genus Rubus, knowledge of their genetics and genome is very limited. Rubus spp. Var. Loch Ness is a high yielding tetraploid $(4 n=28)$ blackberry, and one of the most widely cultivated varieties. However, and despite its high added economic value and as a source of bioactive compounds, its genome has not been sequenced yet. Therefore, other strategies need to be used to gain knowledge to improve production and health related benefits.

RNA-Seq has been recognized as a powerful tool to analyze the transcriptome of an organism that has not been completely sequenced (Wang et al., 2009). The sequencing of RNA has long been recognized as an efficient method for gene discovery and remains the gold standard for annotation of both coding and non-coding genes (Adams et al., 1991). Furthermore, the RNASeq method offers a holistic view of the transcriptome (Wang et al., 2010), and the information obtained from this wide analysis can be used to study specific pathways or physiological situations in a given species, in order to obtain useful information.

Many studies have addressed flavonoid synthesis, identification and subcellular accumulation mechanisms in different plant species as in grapevine (Perez-Diaz et al., 2016), soybean (Dastmalchi et al., 2017), banana (Dong et al., 2016) among others (Zhao, 2015) resulting in characterization of many specific transporters in different organs and accurate flavonoid profiles. Long distance transport has been demonstrated in $A$. thaliana (Buer et al., 2007) And the possibility of long distance flavonoid translocation in grapevine has been suggested, based on the presence of a specific transporter on the vascular bundles, which expression increases from veraison to harvest (Petrussa et al., 2013). Most studies on blackberry and other berries (Paredes-López et al., 2010) have been done on fruits since they are the edible part and therefore, hold the health-potential. The biosynthesis of flavonols and anthocyanins in blackberry fruits along maturation has already been described, demonstrating an increased biosynthesis of anthocyanins in the black ripen stage (Garcia-Seco et al., 2015). On the other hand, it may be anticipated that blackberry leaves also have an active secondary metabolism, in which flavonols must be abundant to participate in adaptation to environmental changes.

Based on this background, the aim of this study is focused on (i) the relevance of the transcriptome to envisage the specific situation of leaves and red fruits at the same time point, and (ii) to establish the relationship of the flavonol-anthocyanin pathway core and regulatory gene expression and their contents in two different organs, given the relevance of these metabolites for defense in leaves and for human health in fruits. In this work, we describe the transcriptome of leaves and red fruits by alignment to Fragaria as a reference genome sequence from the same family (Rosaceae); then, both transcriptomes are compared, quantification of core and regulatory genes of the flavonolanthocyanin pathway expression by RT-qPCR, and HPLC-MS analysis of leaves and fruits is carried out. Differential gene expression suggests that leaf-flavonols are translocated to fruits during the early stages of maturation to achieve the high amounts accumulated therein on ripe fruits.

\section{MATERIALS AND METHODS \\ Plant Materials, Growth Conditions, and RNA Extraction}

The Rubus spp. cv. Loch Ness plants used in this study were kindly provided by Agricola El Bosque S.L. "La Canastita" (Lucena del Puerto, Huelva, Spain). Plants and greenhouses were handled according to regular agricultural practices (RamosSolano et al., 2014). Plants were grown in Huelva (South Eastern Spain) from September 2014 to February 2015 under "winter cycle." Shortly, plants undergo an artificial cold period before transplant to greenhouses in order to start their regular cycle. Blackberry cycle has three stages: vegetative, flowering and flowering-fruiting; the duration of these stages is variable depending on the transplant moment, and each average one third of the plant's life. In this experiment, plants were transplanted at the end of September, flowering in November and fruiting in January when flowers, green, red and black fruits are present in the plants at the same time. Fruits were sampled in red stage. Leaves and fruits were immediately frozen with liquid nitrogen and brought to the lab. Three replicates were taken, being each one constituted from plant material of 20 plants; a total of 25 leaves and $125 \mathrm{~g}$ of ripening fruits were randomly sampled and pooled constituting the replicates.

Prior to RNA extraction, samples were removed from the $-80^{\circ} \mathrm{C}$ freezer and grounded to a fine powder with liquid nitrogen using a sterilized mortar and pestle. Total RNA was isolated from each replicate (red fruits and leaves) with Plant/Fungi Total RNA Purification kit (50) $\left(\right.$ NORGEN $^{\mathrm{TM}}$ ) (DNase treatment included) and after confirmation of RNA 
integrity using Nanodrop ${ }^{\mathrm{TM}}$ and Experion ${ }^{\mathrm{TM}}$, total RNA was sent to Exiqon ${ }^{\mathrm{TM}}$ for sequencing.

\section{RNA-Seq \\ Quality Control and Library Preparation}

RNA obtained from the three biological replicates were pooled and separated in three analytical replicates for RNA-Seq analysis. RNA samples were DNase treated and extracted as described before. Thirty microliters of RNA samples were passed through quality control with Nanodrop ${ }^{\mathrm{TM}}$ and Experion ${ }^{\mathrm{TM}}$, after that total RNA meeting quality criteria was sent to Exiqon ${ }^{\mathrm{TM}}$ for sequencing. A total of six libraries were done, three form leaves and three from fruits.

During the library preparation, poly-A tailed transcripts were enriched, as mRNA sequencing targets this type of transcripts, using an Oligo-dT magnetic bead-based system were enriched. The poly-A tailed transcripts include the coding mRNAs (1$4 \%$ of the whole transcriptome), so by this enrichment the appropriate depth of the sequencing for coding mRNA was achieved. The library preparation also retains information of which of the two strands of DNA was used to transcribe the given RNA, which enables the detection of antisense transcript expression. Mitochondrial poly-A tailed transcripts were bioinformatically filtered since they were considered to be high abundance sequences. The sequencing was paired end, which increases the mapping percentage to poorly annotated genomes, and identifies splice variants.

Two types of sequencing library quality controls were performed, firstly after the library preparation and bead based size selection, the size distribution of the library was evaluated using a Bioanalyzer high sensitivity DNA chip. Then, qPCR based quantification of each library was performed, and samples were normalized and pooled in equimolar ratios. After pooling of sample libraries, qPCR based quantification was performed on the library pool to ensure optimal concentration for cluster generation on the flow cell.

Mapping of the sequencing data represents a useful quality control step in the Next generation sequencing (NGS) data analysis pipeline as it can help to evaluate the quality of the samples. For this purpose, reads were classified in the following classes (i) outmapped reads or high abundance reads: rRNA, mtRNA, polyA, and PolyC homopolymers; (ii) unmapped reads: no alignment to reference genome possible; (iii) mappable reads: alignment to reference genome possible. In a typical experiment it is possible to align $60-90 \%$ of the reads to the reference genome. However, this number depends upon the quality of the sample and the coverage of the relevant reference genome; if the sample is degraded, fewer reads will be mRNA specific and more material will be degraded rRNA. In this case where the reference genome is different from the host organism, the mapping fraction is expected to be significant lower. To test the differences between the blackberry and strawberry, we have taken reads that align to blackberry contig sequences, these reads should thus be bona fide blackberry sequences, and aligned them to the strawberry genome. Following this procedure, $30 \%$ of the reads are expected to be mapped to strawberry genome with the relatively loose stringency settings.

\section{Sequencing}

The library pools to be sequenced were denatured and diluted/neutralized in the required concentrations. Then, cluster generation was performed on the appropriate flow cell using single molecule clonal amplification. Finally, the highthroughput NGS was performed using the Illumina sequencing technology platform.

\section{Transcriptome Aligned}

The raw data (cDNA) was aligned to the reference sequence, using as reference the sequence of wild strawberry (Fragaria vesca subesp. Vesca) a species of same family (Rosaceae), but from a different genus, Fragaria.

The strawberry genome and gene information were downloaded from the Washington State University and Clemson University website (http://www.rosaceae.org), funded by the 2009 USDA NIFA Specialty Crop Research Initiative Program.

\section{Annotation and Classification of Genes}

Raw data ( 75 bp reads) was generated by Exiqon ${ }^{\mathrm{TM}}$ and used for the annotation, aligning the reads against the reference genome of the Strawberry, Fragaria vesca Whole Genome v2.0.a1 Assembly (Annotation reference: Fragaria vesca Whole Genome v2.0.a1 Assembly \& Annotation; The strawberry genome and annotation was found at: https://www.rosaceae. org/species/fragaria_vesca/genome_v2.0.a1). By this method the identification of the genes was achieved.

Genes were then used for BLAST searches and annotation against NCBI $\mathrm{Nr}$ protein database (NCBI non-redundant sequence database). Blast was performed by CloudBlast, using Blastx-fast Blast Program, Non-redundant protein sequences (nr) from 17.01.2016 as Blast DB (Blast database), with a blast expectation value of $1 \times 10^{-10}$, a word size of 6 , and a HSP length cutoff of 33. Gene sequences were further aligned by BLASTX to protein databases such as Swiss-Prot, and KEGG, retrieving proteins with the highest sequence similarity with the given genes along with the functional annotations for their proteins. If results of different databases conflicted, a priority order of $\mathrm{Nr}$, Swiss-Prot, and KEGG was followed. For everything previously described, Blas2 $\mathrm{GO}^{\circledR}$ was used (Conesa and Gotz, 2008). The Blas2 $\mathrm{GO}^{\circledR}$ program was also used to obtain $\mathrm{GO}$ annotations for the genes. The Blast2GO software was then used to perform mapping and annotation (mapping is used to look for associated GO terms to the Hit Blast, and annotation selected from this GOs those with good statistical support). Annotation was made with an annotation cutoff of 55 , and a GO weight of 5 .

\section{Expression Levels}

Gene expression levels were calculated using Fragments Per Kilobase of transcript per million Mapped reads (FPKM) method. The formula used is FPKM $=10^{9} * \mathrm{C} /(\mathrm{N} * \mathrm{~L})$, where $\mathrm{C}$ is the number of mappable reads that fell onto the gene's exons, $\mathrm{N}$ the total number of mappable reads in the experiment and $\mathrm{L}$ the number of base pairs in the exon. The $\log _{2}$ (fold-change) is the log-ratio of a gene's or a transcript's expression values in two different conditions. The $\log _{2}$ fold change is used to confirm the 
significance of the differential expression between the different samples $\left[\log _{2}\right.$ (fold change) $\geq 1$ (overexpression in fruit), or $\log _{2}$ (fold change) $\leq-1$ (overexpression in leaves)] .

\section{RT-qPCR Analysis of Flavonols and Anthocyanins}

In order to confirm the expression obtained in the RNA-Seq data, a RT-qPCR was performed. The retrotranscription was performed using iScript tm cDNA Synthesis Kit (Bio-Rad). All retrotranscriptions were performed using an GeneAmp PCR System 2700 (Applied Biosystems): $5 \mathrm{~min} 25^{\circ} \mathrm{C}, 30 \mathrm{~min} 42^{\circ} \mathrm{C}, 5$ min $85^{\circ} \mathrm{C}$, and hold at $4^{\circ} \mathrm{C}$. The amplification were performed with a MiniOpticon Real Time PCR System (Bio-Rad): 3 min at $95^{\circ} \mathrm{C}$ and then 39 cycles consisting of $15 \mathrm{~s}$ at $95^{\circ} \mathrm{C}, 30 \mathrm{~s}$ at $50^{\circ} \mathrm{C}$ and $30 \mathrm{~s}$ at $72^{\circ} \mathrm{C}$, followed by melting curve to check the results. To describe the expression obtained in the analysis, cycle threshold (Ct) was used. Standard curves were calculated for each gene, and the efficiency values ranged between 90 and 110\%.Two reference genes were used, actin for fruits and histone for leaves. A different reference gene was used for each organ, since no common gene was found meeting criteria for a reference gene. However, these genes have similar Ct, so they can be compared. Core and regulatory genes studied and the primers used for each appear in Table 1.

To evaluate RT-qPCR values, one-way ANOVA was performed to compare core gene expression and transcription factors in the different organs, using Statgraphics plus 5.1 for Windows. Prior to ANOVA analysis homoscedasticity and normality of the variance was checked with Statgraphics plus 5.1 for Windows, meeting requirements for analysis.

\section{Phenolics and Flavonoids Characterization HPLC-MS Phenolics and Flavonoids Characterization Standards and solvents}

Phenolic acids including, gallic acid, caffeic acid, ferulic acid, and chlorogenic acid were purchased from Sigma (St. Louis, $\mathrm{MO}, \mathrm{USA}$ ) and flavonoids including, kaempferol, kempherol3-O-rutinoside, kempherol-3-O-glucoside, quercetin, quercetin3-O-rutinoside, quercetin-3-O-glucoside, (+)-catechin, (-)epicatechin and cyanidin-3-O-glucoside, were purchased from Sigma and from Extrasynthese Co. ${ }^{\mathrm{TM}}$ (Geney, France).

The standard solutions (10 ppm) were prepared in methanol. All the solvents, as methanol and acetonitrile (Honeywell Riedelde Haen), were LC-MS grade. Purified water was obtained from Milli-Q Plus ${ }^{\mathrm{TM}}$ System from Millipore (Milford, MA, USA). Formic acid was purchased from Aldrich (St. Louis, MO, USA).

\section{Sample preparation}

The extraction of phenolics was performed as follows: $300 \mathrm{mg}$ of powder were added to $300 \mu \mathrm{L}$ of methanol. The mixture was vortexed for $2 \mathrm{~min}$, sonicated for $5 \mathrm{~min}$ and centrifuged at $3,500 \mathrm{rpm}$ for $5 \mathrm{~min}$ at $4^{\circ} \mathrm{C}$. The supernatants were then collected and stored at $-20^{\circ} \mathrm{C}$ until use for LC/MS analysis, and then the extracts were diluted in methanol solution at a ratio of $1: 10(\mathrm{v} / \mathrm{v})$.

\section{LC/MS Analysis}

Phenolic acids and flavonoids were analyzed by LC-MS-IT analysis. The identification of compounds was performed using an Agilent 1100 HPLC system (Agilent Technologies) connected to a Bruker Daltonics esquire $3,000^{\text {plus }}$ ion trap mass spectrometer (Bruker Daltonics) with an electrospray interfase (ESI).

Separation was on a Kinetex XB-C18 column $(100 \times$ $2.1 \mathrm{~mm}, 2.6 \mu$; Phenomenex) running the following gradient of acetonitrile (solvent $\mathrm{B}$ ) vs. $0.1 \%$ aqueous formic acid (solvent $\mathrm{A}$ ): from 0.0 to $1.0 \mathrm{~min} 2 \% \mathrm{~B}$, from 1.0 to $6.0 \mathrm{~min} 10 \% \mathrm{~B}$, from 6.0 to $26.0 \mathrm{~min} 30 \% \mathrm{~B}$, from 26.0 to $36.5 \mathrm{~min} 90 \% \mathrm{~B}$, from 36.5 to 37.5 $90 \% \mathrm{~B}$, from 37.5 to $38.0 \mathrm{~min} 2 \% \mathrm{~B}$, and from 38.0 to $45.0 \mathrm{~min}$ $2 \% \mathrm{~B}$, with a flow rate at $0.3 \mathrm{~mL} / \mathrm{min}$ and column temperature at $60^{\circ} \mathrm{C}$ Peaks were detected by UV/visible light absorbance, collecting chromatograms at $260 \mathrm{~nm}$.

The data acquisition software employed was Esquire Control 5.2. Data analysis was performed using the Data Analysis 3.1 software (Bruker Daltonics) and were adquired in negative and positive ionization mode with the following experimental conditions: nitrogen gas temperature: $350^{\circ} \mathrm{C}$; drying gas flow rate: $11.5 \mathrm{~L} / \mathrm{min}$ and capillary voltage: $\pm 4000 \mathrm{~V}$; nebulizing pressure: 25 psi. Mass spectra were recorded using the full scan mode in the range of $\mathrm{m} / \mathrm{z} 50-1,300$.

\section{Total Phenols}

Total phenols were quantitatively determined with FolinCiocalteu agent (Sigma. Aldrich, St Louis, MO) by a colorimetric method described by $\mathrm{Xu}$ and Chang (2007) with some modifications, gallic acid was used as standard (Sigma-Aldrich, St Louis, MO). One milliliter of extract was mixed with 0.250 $\mathrm{mL}$ of Folin-Ciocalteu 2N (Sigma. Aldrich, St Louis, MO) and $0.75 \mathrm{~mL}$ of $\mathrm{Na}_{2} \mathrm{CO}_{3} 20 \%$ solution. After $30 \mathrm{~min}$ at room temperature, absorbance was measured at $760 \mathrm{~nm}$ with an UVVisible spectrophotometer (Biomate 5). A gallic acid calibration curve was made $(r=0.99)$. Results are expressed in mg of gallic acid equivalents per $100 \mathrm{~g}$ of fresh weigh (FW). All samples were measured in triplicate.

\section{Total Flavonols}

Total flavonols were quantitatively determined through the test described by Jia et al. (1999), using catechin as standard (SigmaAldrich, St Louis, MO). One milliliter of extract was added to a flask of $10 \mathrm{~mL}$ with $4 \mathrm{~mL}$ of distilled water. After that $0.3 \mathrm{~mL}$ of $\mathrm{NaNO}_{2} 5 \%$, and $0.3 \mathrm{~mL}$ of $\mathrm{AlCl}_{3} 10 \%$ were added after $5 \mathrm{~min}$. One minute later, $2 \mathrm{~mL}$ of $\mathrm{NaOH} 1 \mathrm{M}$ were added, and distilled water was added till $10 \mathrm{~mL}$ of total volume. The solution was mixed and measured at $510 \mathrm{~nm}$ with an UV-Visible spectrophotometer (Biomate 5). A catechin calibration curve was made $(r=0.99)$. Results are expressed as $\mathrm{mg}$ of catechin equivalents per $100 \mathrm{~g}$ of fresh weigh (FW). All samples were measured in triplicate.

\section{Total Anthocyanins}

Total anthocyanins were quantitatively determined through the pH differential method described by Giusti and Wrolstad (2001). Extracts were diluted in $\mathrm{pH} 1$ buffer $(0.2 \mathrm{M} \mathrm{KCl})$ and $\mathrm{pH} 4.5$ $\left(1 \mathrm{M} \mathrm{CH} \mathrm{CH}_{3} \mathrm{COONa}\right)$ in 1:15 proportion. After that, absorbance 
TABLE 1 | Primers designed to RT-qPCR expression analysis (Garcia-Seco et al., 2015).

\begin{tabular}{|c|c|c|}
\hline Gene & Forward primer & Reverse primer \\
\hline Ru4CL & 5'CCAGAAGTTCGAGATCAACAAGT & 5'GTCGGGACACTTGGTAATAGACA \\
\hline RUACT & 5'ATGTTCCCTGGTATTGCAGAC & 5'CCACAACCTTGATCTTCATGC \\
\hline RUANR & 5'TCGCAATGTACTTCCAAGAAAC & 5'CTTCATCAGCTTACGGAAATCAC \\
\hline RUANS & 5'TTGGTCTGGGATTAGAAGAAAGG & 5'CTGAGGGCATITTGGGTAGTAAT \\
\hline $\mathrm{RuC4H}$ & 5'CATCTGTAGGGAAGTGAAGGAGA & 5'ACTTCAACCCTTCGTTAGTTGTG \\
\hline $\mathrm{RuCH} / 1$ & 5'CAAGAAGGATTCCATCATCACA & 5'CTCCACTITGATCTITGACGACT \\
\hline $\mathrm{RuCH} 2$ & 5'GAGGCAGTTCTTGAGTCAATCAT & 5'CACGCTATCATCACTCACTITCA \\
\hline $\mathrm{RUCHS}$ & 5'ATGGTGGTTGTTGAAATTCC & 5'CTGGATTGCACACCCAGGTGGCCC \\
\hline$R U D F R$ & 5'AATCAGAAGAAGGTGAAGC & 5'CATTAKSACAAGTITGGTG \\
\hline$R u F 3^{\prime} 5^{\prime} H$ & 5'ATGCCHCATGYYDCCTTAGCHAAAATGG & 5'TGGGCAATHGGRMGAGAYCC \\
\hline $\mathrm{RuF3H}$ & 5'ATGGCTCCTACACCTACTAC & 5'TGGATCACCGTTCAACCTGTGGAAGG \\
\hline$R u F 3^{\prime} H$ & 5'CCTATCTCCAAGCTGTCATCAAG & 5'GTGGTATCCGTTGATTTCACAAC \\
\hline RUFLS & 5'CCTACAGGGAAGTCAATGAGAAA & 5'CACATGGGATTTCAGTACCTTCT \\
\hline RUGST1 & 5'TACTAGAATCACAAGCACCAGCA & 5'ACCCAAAACTCACATAGACAACG \\
\hline RUGST2 & 5'GAACTCATTGCTTGAGAGCGTAG & 5'GATCTTCCACACTTCCTCTACCA \\
\hline RuLAR & 5'GTGGAGTCCCATACACGTACATT & 5'CTGAAACTGATCTAACGGTGGAA \\
\hline RUPAL1 & 5'GAGGAACTGGGGACTAGTITGTT & 5'AGCAGAGGATCAATCAGCTITC \\
\hline RUPAL2 & 5'GACTTGCTCTTGTTAATGGCACT & 5'GAAAATCGCAGACAAGATTTCG \\
\hline RUMYB1 & 5'CTCATTGACAGGAACAGGTGTC & 5'CCTACAACAACACCAACGAGAAT \\
\hline RUMYB3 & 5'GAGCTGTAGGTTAAGATGGACAAA & 5'GTाCCAAGGATAGAATGGAGATG \\
\hline RUMYB4 & 5'ACAGCTCAGGACTCTGCTACAAC & 5'GGTIATAGACTCTITGCCCACA \\
\hline RUMYB5 & 5'ACTCAATCCAGACTCCTCATCTG & 5'AGGAAGTGATTGGACTITAGGG \\
\hline RUMYB6 & 5’TCCTATGGAGTACTTCCAAGCTC & 5'TATGGCTGTITAGTCCTCCTTGA \\
\hline RUTTG1 & 5'GTTGTGATCTTGGATATCCGTTC & 5'GAACAAATGTGCCTATAACTCTGC \\
\hline
\end{tabular}

was measured at 520 and $720 \mathrm{~nm}$ respectively, in a UVVisible spectrophotometer (Biomate 5 ). A cyanidin-3-glucoside calibration curve was made $(r=0.99)$. Results are expressed in cyanidin-3-glucoside equivalents per $100 \mathrm{~g}$ of fresh weigh (FW). All samples were measured in triplicate.

\section{RESULTS}

\section{Transcriptome Sequencing and Assembly}

To construct a de novo transcriptome database, six mRNA libraries were generated by Illumina sequencing, three from leaves and three from red fruits of Rubus spp. cv. Loch Ness.

Table 2 summarizes mapping results. In addition to these, it also shows the total number of reads obtained for each sample. On average, 57 million reads (75 bp read length) were obtained from each sample and genome mapping was on average $26 \%$ for all samples. The uniformity of the sample's mapping indicates that samples are comparable.

In a typical experiment it is possible to align $60-90 \%$ of the reads to the reference genome, however, this number depends upon the quality of the sample and the coverage of the relevant reference genome; if the sample is degraded, fewer reads will be mRNA specific and more material will be degraded rRNA. In this case, in which the reference genome is different from the host organism, the mapping fraction was expected to be significantly lower. We found that $26 \%$ of the reads could be mapped to strawberry genome with relatively loose stringency
TABLE 2 | Total reads of the three different replicates from fruit and leaves.

\begin{tabular}{lccccc}
\hline $\begin{array}{l}\text { Sample } \\
\text { name }\end{array}$ & $\begin{array}{c}\text { Total } \\
\text { readcount }\end{array}$ & $\begin{array}{c}\text { rRNA } \\
\text { (\%) }\end{array}$ & $\begin{array}{c}\text { Other } \\
\text { (mtRNA) }\end{array}$ & $\begin{array}{c}\text { Mapped } \\
\text { (\%) }\end{array}$ & $\begin{array}{c}\text { Unmapped } \\
\text { (\%) }\end{array}$ \\
\hline Fruit 1 & 65264074 & 0.001 & 0.019 & 25.399 & 74.598 \\
Fruit 2 & 52300955 & 0.001 & 0.017 & 25.499 & 74.498 \\
Fruit 3 & 42196474 & 0 & 0.014 & 25.199 & 74.798 \\
Leaves 1 & 43184377 & 0 & 0.138 & 26.374 & 73.528 \\
Leaves 2 & 43979951 & 0 & 0.174 & 25.671 & 74.216 \\
Leaves 3 & 49606567 & 0.001 & 0.303 & 27.359 & 72.492 \\
\hline
\end{tabular}

Total Readcount, \%rRNA, \% other RNA, \% Mapped RNA, and \% Unmapped RNA.

settings. Mapping Alignment of paired-end reads to the genome was carried out against the Fragaria vesca Whole Genome v2.0.a1. The strawberry genome and annotation was found at: https://www.rosaceae.org/species/fragaria_vesca/genome_v2.0.a1. A total of 285 genes were $>1,000 \mathrm{bp}$ in length, and 30,001 were $<1,000$ bp (Figure 1).

After sequencing and mapping alignment, normalized expression and differential expression (fruit vs. leaves) identified a total of 33,666 genes. The heatmap diagram (Figure 2) shows the result of the two-way hierarchical clustering of RNA transcripts and samples. It includes the 500 genes that have the largest coefficient of variation based on FPKM counts. Each row represents one gene and each column represents one sample. The color represents the relative expression level 


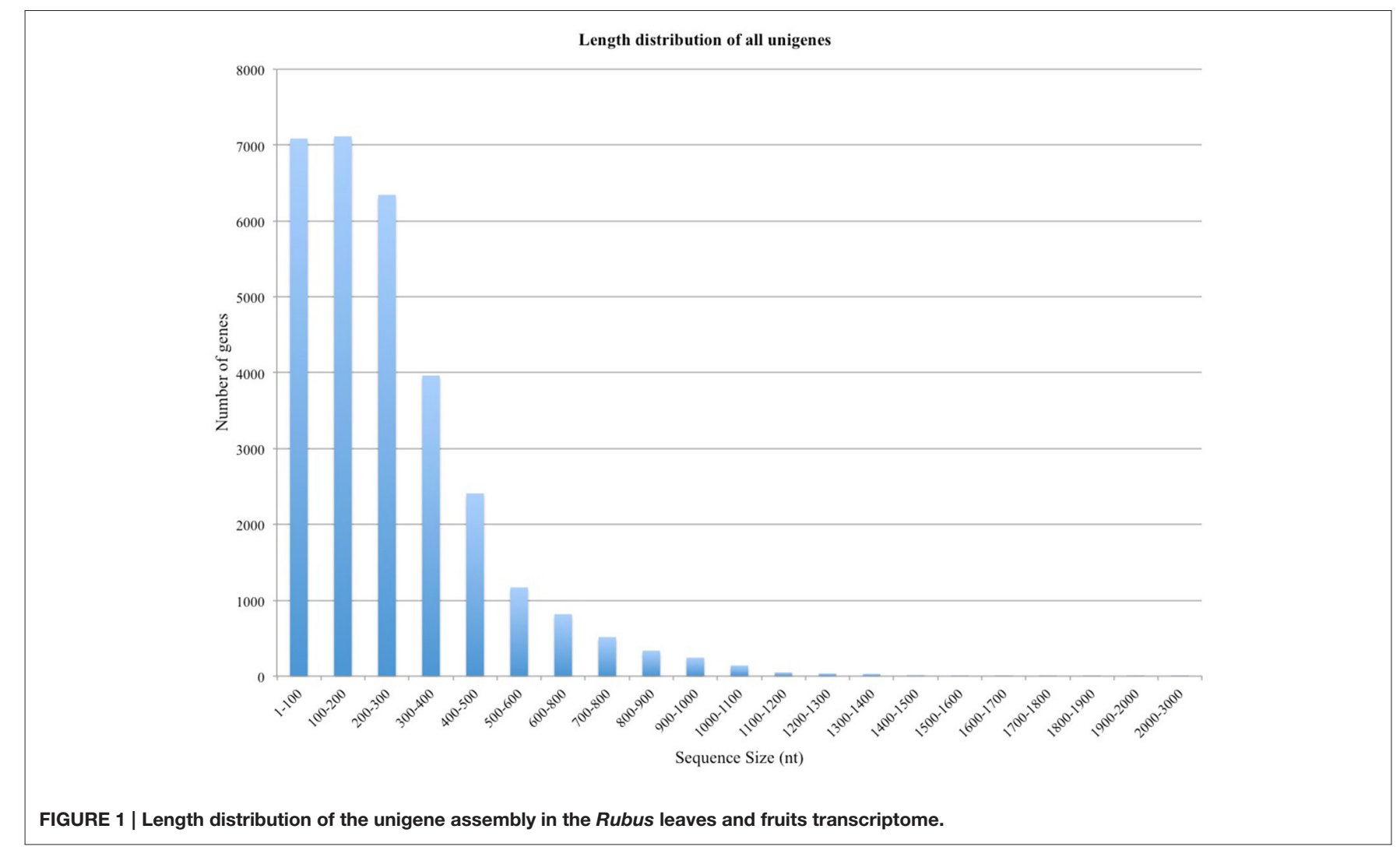

of a transcript across all samples. The color scale is shown below: red represents an expression level above the mean; green represents an expression level below the mean. When tissues were compared, the expression pattern showed that 20,463 genes were common to both organs (expression without significant differences), and 6,604 genes were significantly overexpressed in fruit, while 6,599 genes were significantly overexpressed in leaves (Figure 3).

\section{Blast, Mapping, Annotation, and Interproscan}

Once the data was filtered, Blast2Go was used to perform the blast, mapping, annotation, and InterProScan. The InterProScan analysis gives a functional analysis of proteins, classifying them in families, and predicting important sites and domains. InterProScan analysis indicates that the Cytochrome P450 family is very abundant in leaves (Figure 4) and fruits (Figure 5). Also relevant the presence of glycosiltransferases, that appear in second position in both organs, and a general sugar transporter in leaves in fourth position. InterProScan analysis of common genes is shown in supplementary material.

GO analysis (Figure 6) shows that the most abundant genes are grouped in metabolic and cellular processes as well as binding, and catalytic activity. GO analysis for leaves and fruits is shown in independent figures in supplementary material.

A detailed analysis of overexpressed genes in fruits (supplementary material) indicate presence of terpenes, a very low presence of alkaloids while phenolic compounds predominate.

Overexpressed genes in leaves are related to an active photosynthesis (mostly related to photosystems I and II), and to an efficient capacity of ROS scavenging, shown in the high number of transcripts of SOD, ascorbate oxidase and glutathione reductase. Also, glutamate and glutamic acid metabolism-related transcripts are abundant in leaves as well as auxin-related genes. As regards of secondary metabolism, leaves show similar expression patterns as in fruits: alkaloid metabolism is almost absent, terpene metabolism is similar, and phenolic compounds metabolism predominate. Different to fruits, leaves show abundant transcripts of the shikimate pathway; interestingly, isoflavones, and anthocyanins-related genes are also overexpressed. Interestingly, although MATE, and $\mathrm{ABC}$ transporters and Glutathione-S-Transferases were expressed in leaves and fruits, only ABC and GST were overexpressed in leaves. Finally, abundant transcripts related to defense (PRs and LRRs) were also found in leaves (supplementary material).

\section{RT-qPCR Analysis of the Flavonol-Anthocyanin Pathway}

The flavonol-anthocyanin pathway is illustrated in Figure 7. Expression of core (Figure 8) and regulatory genes (Figure 9) was studied in leaves and fruits. 


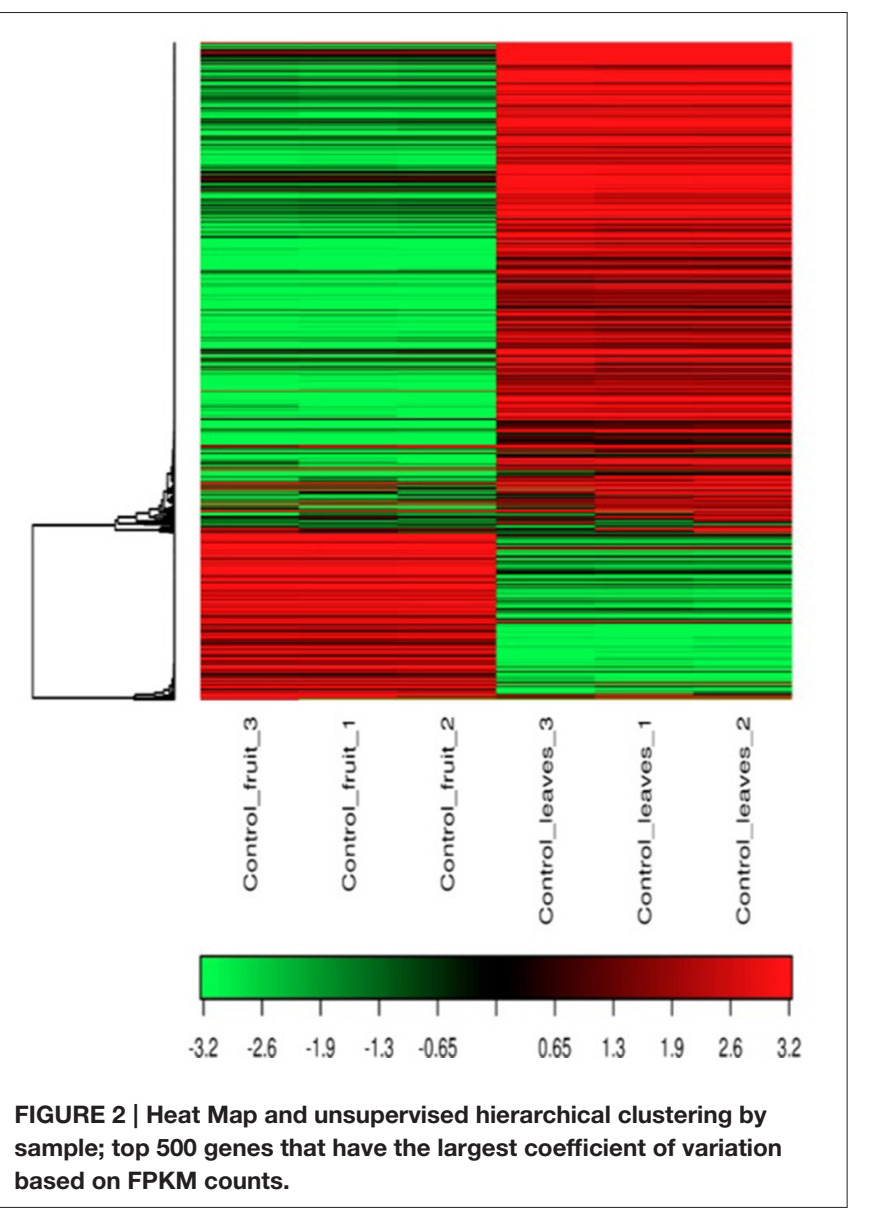

based on FPKM counts.

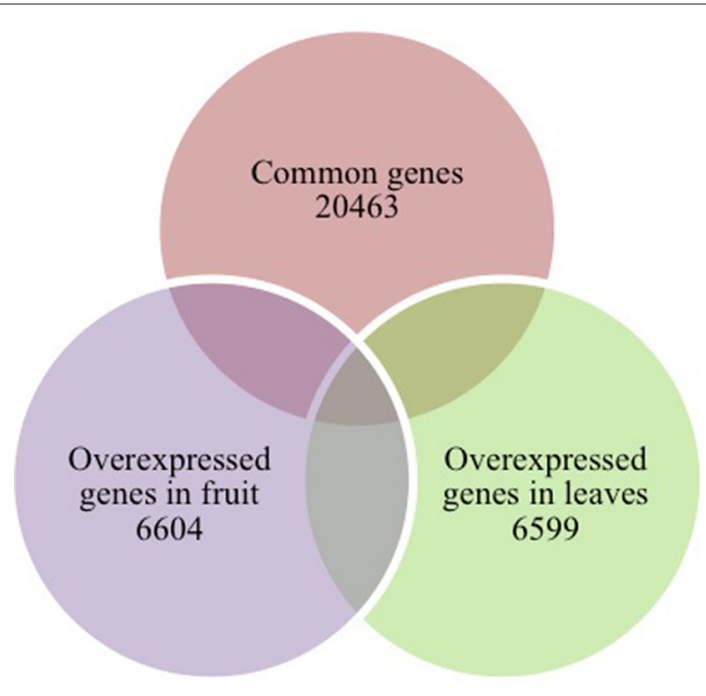

FIGURE 3 | Venn diagram of tissue-specific overexpressed and common genes.

As regards to core genes (Figure 8), all were expressed in leaves and fruits, except for RuANS that was downregulated in both. In general, expression of the flavonol-anthocyanin pathway core genes was higher in leaves. Two isoforms were studied for $R u P A L, R u C H I$, and $R u G S T$. Both $R u P A L$ isoforms were found in both organs, being RuPAL1 significantly overexpressed in fruits. Expression of chalcone synthase $(R u C H S)$ involved in extension of 4 hydroxy-cumaroyl-CoA side chain, showed a significant four-fold expression in leaves as compared to fruits; this expression, coupled to a significant four-fold and two-fold expression of $\mathrm{RuCHI} 2$ and $\mathrm{RuF} 3 \mathrm{H}$ respectively, was consistent with the higher concentration of flavonols found in leaves (Figure 8). RuGST1 was expressed in both organs while RuGST2 was expressed only in leaves, and these differences were significant.

Conversely to core genes, expression levels of all regulatory genes or transcription factors (TF) were higher in fruits (Figure 9).The regulatory gene's expression (Figure 9) showed that $R u M Y B 1, R u M Y B 5$, and RuMYB6 had higher expression in red fruit than in leaves. The negative pathway regulator $R u M Y B 4$, showed high expression in red fruit while leaves showed a lower expression of this RuMYB4, consistent with higher expression of $\mathrm{RuC} 4 \mathrm{H}$ in leaves.

\section{Bioactive Characterization Bioactives Quantification}

Bioactives were quantified by colorimetry (Table 3) and identified by HPLC-MS (Figure 10). Red fruits contained $560 \mathrm{mg}$ gallic acid equivalents (total phenols), $34 \mathrm{mg}$ catechin equivalents (total flavonols), and $108 \mathrm{mg}$ cyanidin-3-glucoside equivalents (total anthocyanins). Leaves showed $1,876 \mathrm{mg}$ gallic acid equivalents, $296 \mathrm{mg}$ catechin equivalents and $55 \mathrm{mg}$ of cyanidin-3-glucoside equivalents. All results are expressed per $100 \mathrm{~g}$ fresh weigh. In summary, contents in leaves as compared to fruits were as follows: total phenolics were three-fold in leaves, while total flavonoids were six-fold in leaves and anthocyanins were two-fold in fruits.

\section{Peak Identification and Assignment}

The LC/MS analysis was performed using defined conditions that provided a good separation profile (Figure 10). Identification and peak assignment was based on comparison of their retention time and mass spectra data with those of standards available, and the results are indicated in Tables 4, 5, where $m / z$ experimental values from the BPC (Base Peak Chromatograms) obtained in negative and positive ion mode as well as the fragments in source are shown.

Due to the lack of commercial standards, most peaks have not been identified and no information about these unknown compounds has been provided. However, MS fragmentation in source has helped us to establish a tentative identification for most of the significant peaks by comparison with bibliography. Thus, caffeoyl-O-hexosides $(R t=1.0-3.3 \mathrm{~min})$ with a $[\mathrm{M}-\mathrm{H}]^{-}$ ion at $m / z 340.9$ and $[\mathrm{M}+\mathrm{Na}]^{+}$and $[\mathrm{M}+\mathrm{K}]^{+}$adducts at $m / z$ 365.0 and $m / z=381.0$ respectively were found. Many caffeic acid-O-dihexoside and other caffeic acid derivatives were also found mainly in leaves ( $R t=20-45 \mathrm{~min}$ ) with $[\mathrm{M}-\mathrm{H}]^{-}$ion at $\mathrm{m} / z$ 503.1, and chlorogenic acid was identified only in fruits (Table 4).

In Blackberry, characteristic flavonols were kaempferol and quercetin derivatives, and cyanidin-3-O-glucoside as the most 


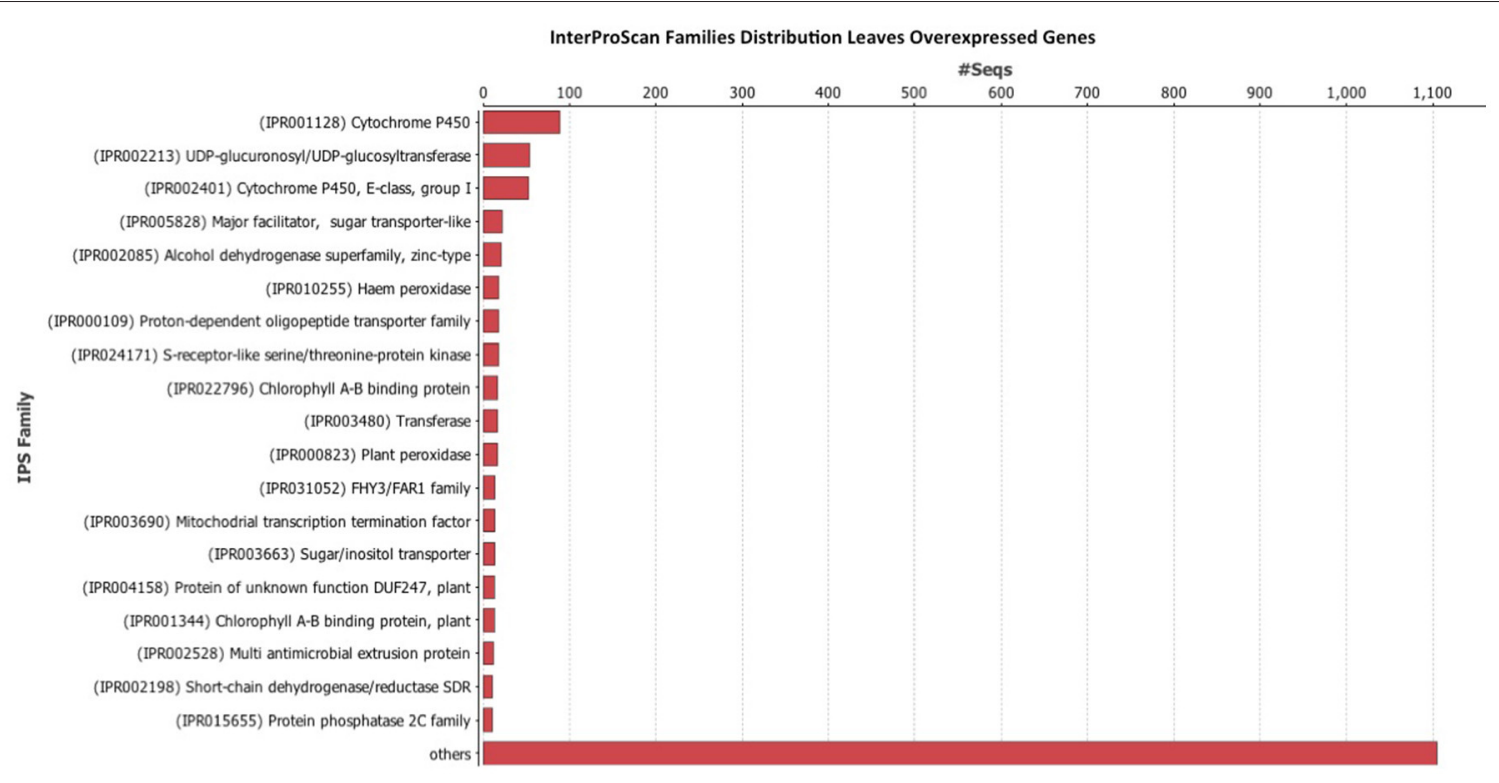

FIGURE 4 | Histogram of InterProScan analysis of Rubus spp. genes in leaves.

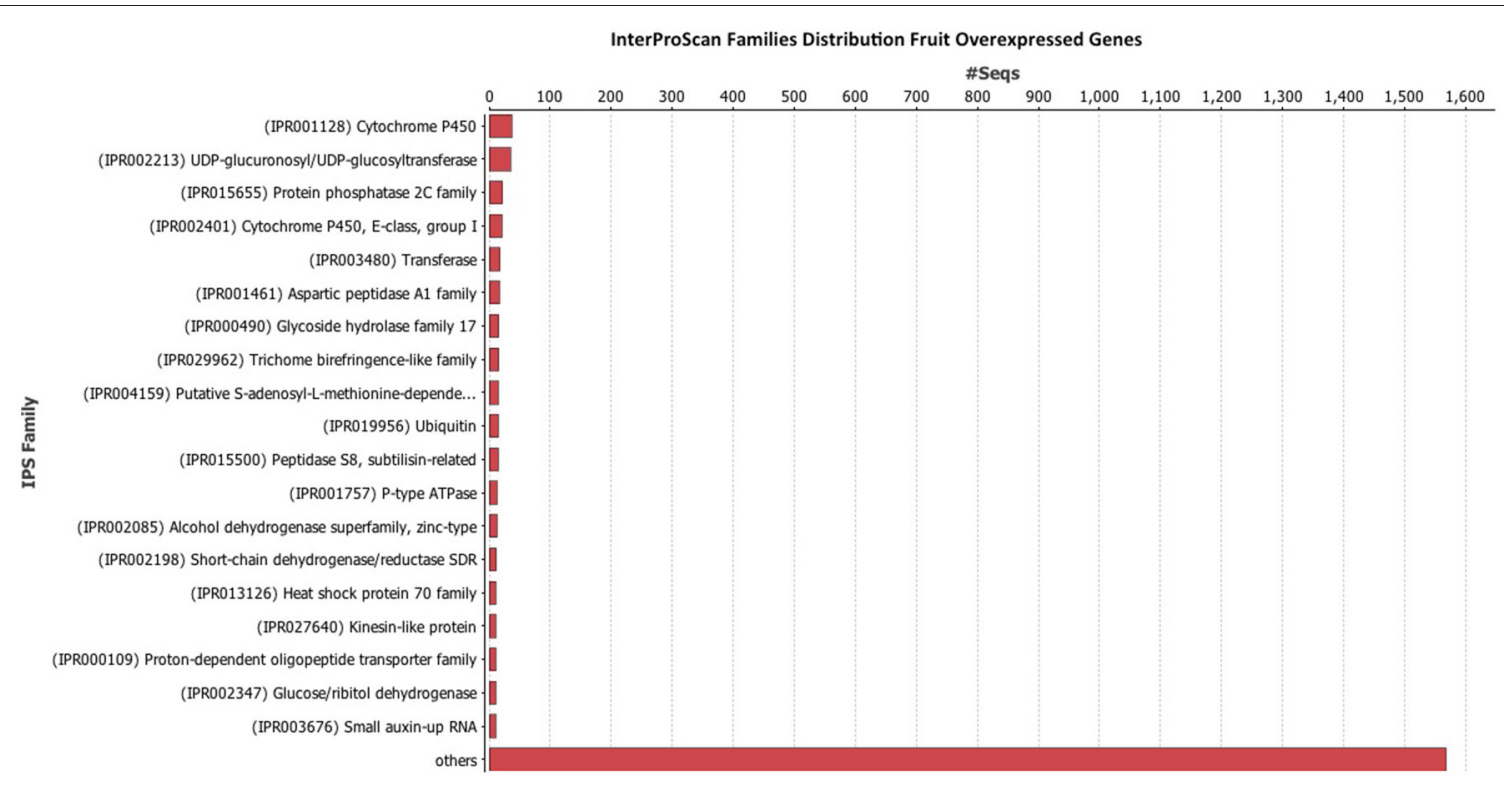

FIGURE 5 | Histogram of InterProScan analysis of Rubus spp. genes in fruit.

abundant anthocyanin, and (+)-catechin and (-)-epicatechin among catechols. However, leaf and fruits showed different profiles. According to LC/MS data (Table 4) (+)-catechin, (-)epicatechin, chlorogenic acid and cyanidin 3-O-glucoside were present in red fruits. Cyanidin-3-O-glucoside was the only compound common to leaves and fruits (Table 5). On the other hand, the flavonols quercetin-3-O-rutinoside kaempferol-3-Orutinoside, and quercetin-3-O-glucoside were identified only in leaves. Interestingly flavonol glucosides were present only in leaves and derivatives of these flavonols, mainly quercetins, were present only in fruits.

\section{DISCUSSION}

Flavonoids are secondary metabolites that play a relevant role in plant defense, being of great importance against biotic (fungus, bacterias, herbivores; Koskimaki et al., 2009; Makoi et al., 2010; Kim et al., 2011) and abiotic stress (light, temperature, water supply, minerals, and $\mathrm{CO}_{2}$; Ramakrishna and Ravishankar, 2011). They are also beneficial compounds for human health, having different and beneficial effects as antioxidants, cardioprotective, antihypertensive, or blood glucose regulation (Lotito and Frei, 2004; Cassidy et al., 


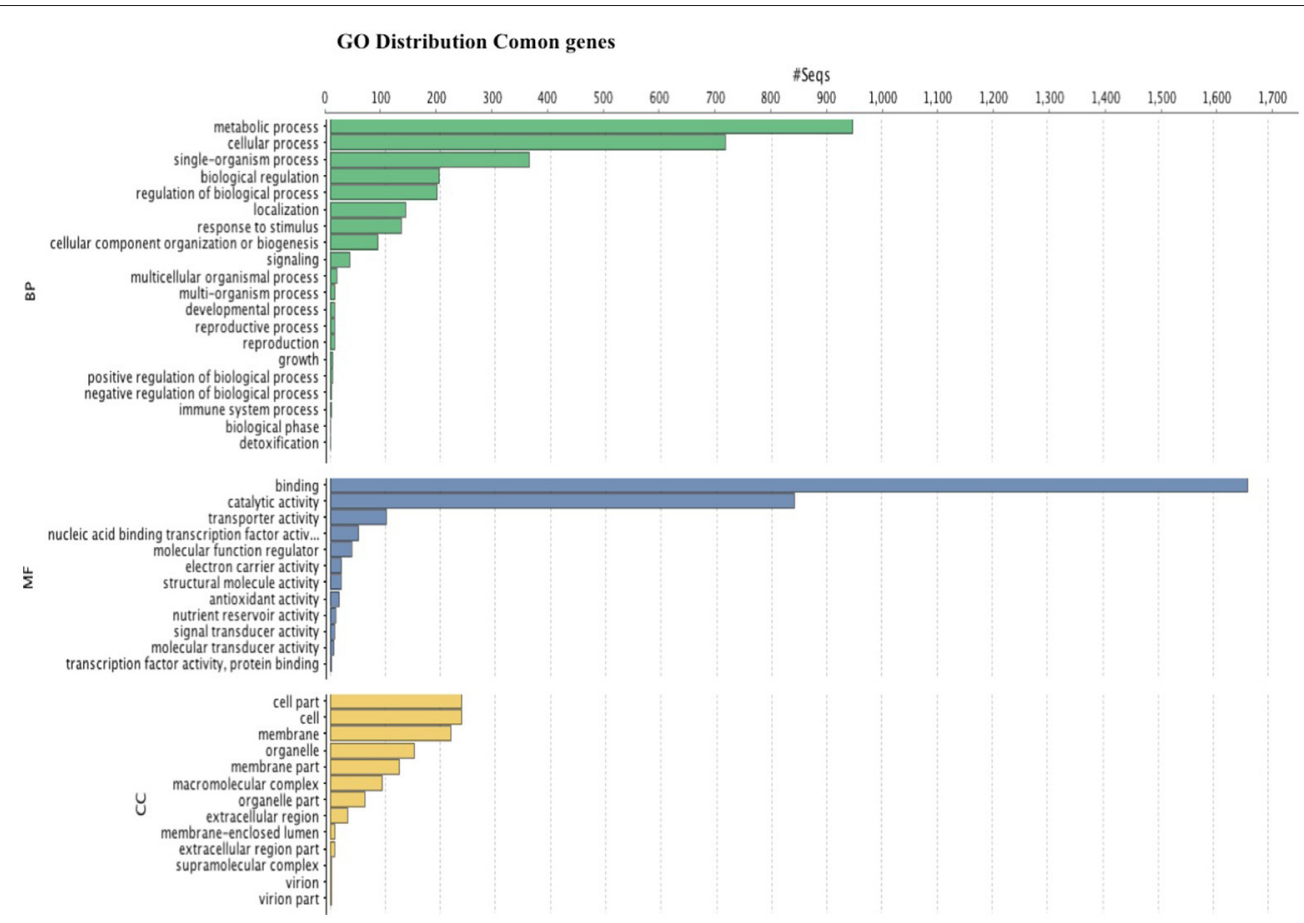

FIGURE 6 | Histogram of GO classifications of Rubus spp. unigenes. Results are summarized for the three main categories: biological process, cellular component and molecular function.

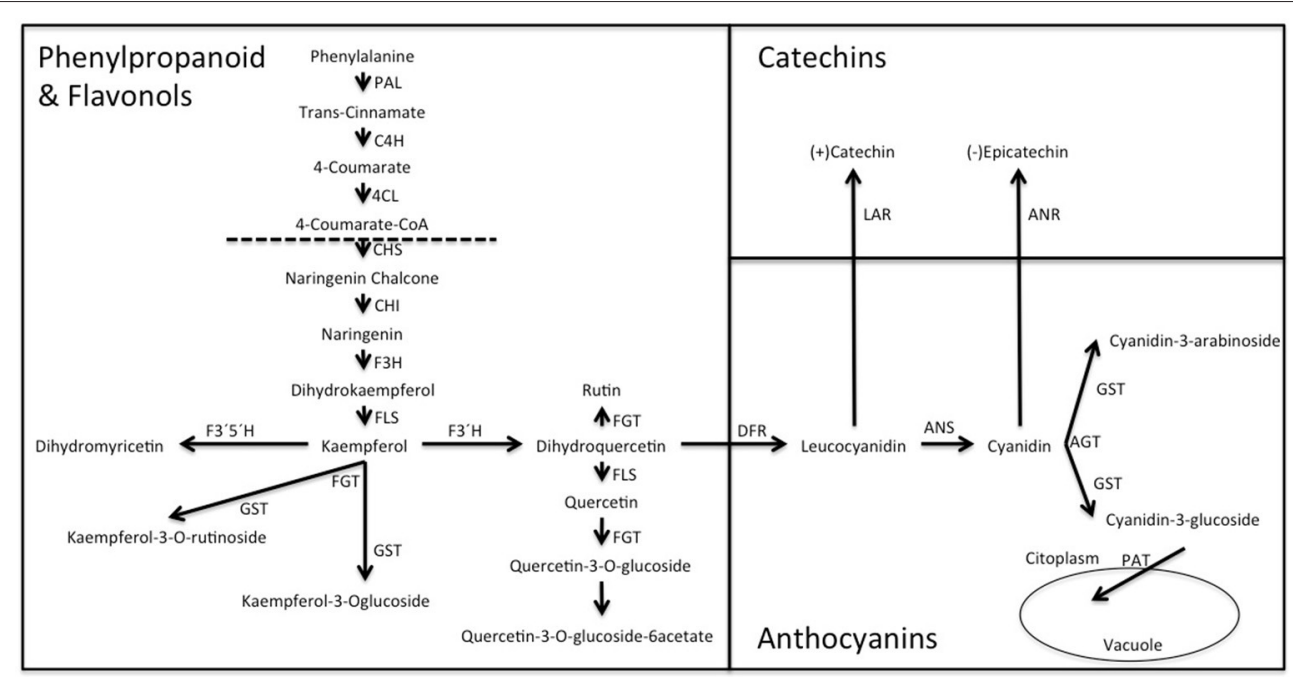

FIGURE 7 | Biosynthesis of anthocyanins, flavonols and catechins via the flavonoid pathway in Rubus spp. Var. Loch Ness. Phenyl alanine ammonio-lyase (RUPAL1 and RUPAL2), Cinammate 4 hydroxylase (RuC4H), 4-coumaryl: CoA ligase (RuACL), Chalcone synthase (RuCHS), Chalcone Isomerase1 (RuCHI1), Chalcone Isomerase2 (RuCHI2), Flavonol-3-hydroxylase (RuF3H), Flavonoid $3^{\prime} 5^{\prime}$ hydroxylase (RuF3' $\left.5^{\prime} H\right)$, Flavonoid $3^{\prime}$ hydroxylase (RuF3'H), Flavonol synthase (RuFLS), Leucoanthocyanidin reductase (RuLAR), Anthocyanidin reductase (RuANR), Dehydroflavonol reductase (RuDFR), Anthocyanidin synthase (RuANS), Glycosiltransferases (RUGST1 and RUGST2).

2011; Martin et al., 2011; Fuchs et al., 2016). Therefore, this study was undertaken to gain knowledge about bioactive metabolism in leaf and fruit, to understand the mechanism by which the plant synthesizes and accumulates bioactives in fruits.
Blackberries were sampled in the maximum production period, therefore, with a very active metabolism (Ramos-Solano et al., 2014); an active photosynthesis is necessary to feed carbon into growing leaves, flowers, and fruits. Consistent with this statement, abundant transcripts related to photosynthesis were 
Flavonol-Anthocyanin Pathway Gene Expresion Fruit

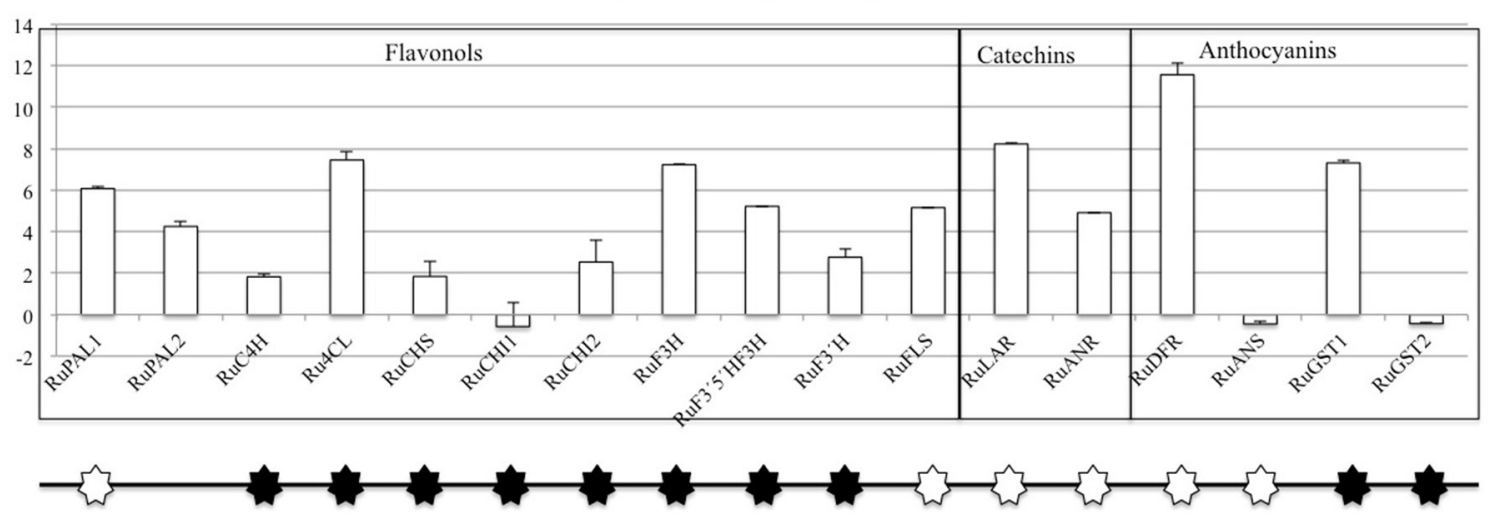

Flavonol-Anthocyanin Pathway Gene Expression Leaves

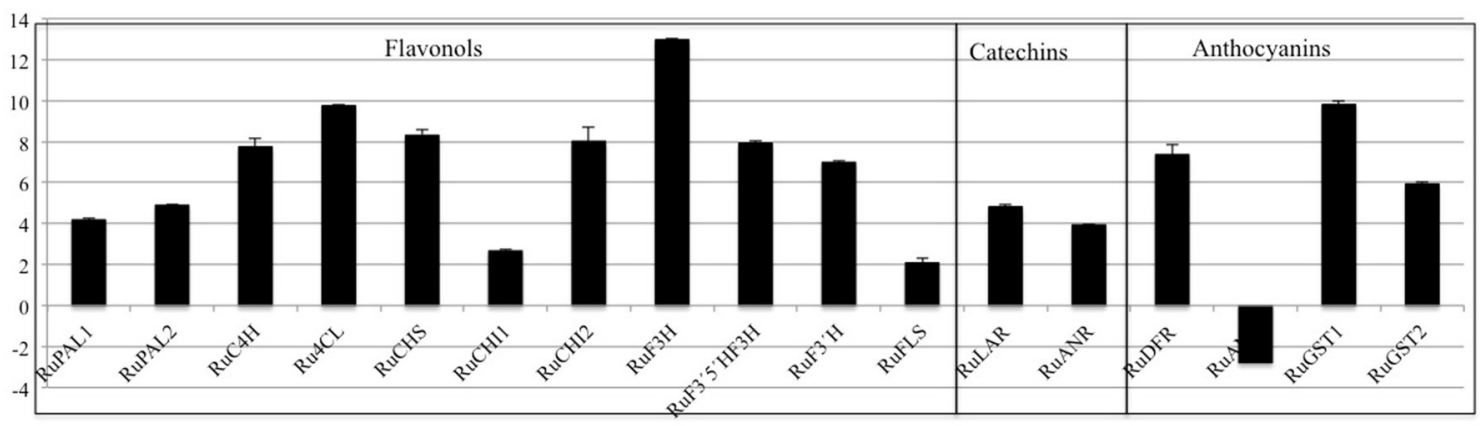

FIGURE 8 | Flavonol-Anthocyanin Pathway gene expression analyzed by RT-qPCR. Phenyl alanine ammonio-lyase (RUPAL1 and RUPAL2), Cinammate 4 hydroxylase $(\mathrm{RuC} 4 \mathrm{H})$, 4-coumaryl-CoA ligase (Ru4CL), Chalcone synthase (RuCHS), Chalcone Isomerase1 (RuCHI1), Chalcone Isomerase2 (RuCHI2),

Flavonol-3-hydroxylase $(R u F 3 H)$, Flavonoid $3^{\prime} 5^{\prime}$ hydroxylase $\left(R u F 3^{\prime} 5^{\prime} H\right)$, Flavonoid $3^{\prime}$ hydroxylase $\left(R u F 3^{\prime} H\right)$, Flavonol synthase $(R u F L S)$, Leucoanthocyanidin reductase (RuLAR), Anthocyanidin reductase (RUANR), Dehydroflavonol reductase (RuDFR), Anthocyanidin synthase (RuANS), Glycosiltransferases (RuGST1 and RuGST2). Asterisks indicate significant differences, according to Fisher test $(p<0.05)$. Black and white asterisks indicate higher expression in leaves or fruits, respectively.

found in leaves, among which are representatives of ferredoxin, which intervenes in the cyclic and acyclic photophosphorylation, as well as transcripts responsible for the formation of proteins of photosystems I and II, and photosynthetic pigments, mainly chlorophylls A and B.

This active photosynthesis supports an active glutamate and glutamic acid metabolism. Related transcripts were abundant in leaves supporting high activity on $\mathrm{N}$ assimilation (Vance et al., 1995), and consistent with plant growth and productivity. The auxin-related genes also support an intense growth activity in the vegetative organs (Went, 1933).

Interestingly, defensive metabolism was also very active, and endochitinases (RuPR4), endoglucanases (RuPR2), endoglucosidases, $(R u P R 1)$, subtilisin, proteases, and thaumatine genes are noticeably expressed, along with other minoritary antimicrobial compounds. This active defensive metabolism is consistent with the powdery mildew outbreak in production greenhouses reported by Agricola El Bosque at that time, and reflects defensive metabolism that enables plant adaptation to environmental conditions.

As regards of secondary metabolism, leaves show similar expression patterns as in fruits: very low alkaloid metabolism, similar terpene metabolism, with a predominance of phenolic compounds metabolism. As secondary metabolites are highly apolar, they usually appear as sugar-bound heterosides, calling for high presence of glycosylating enzymes as well as a high activity related to vacuolar transport (Figures 4, 5), where they are stored (Yauk et al., 2014) as highlighted by Interproscan data (Figures 4, 5).

According to transcript abundance, terpenes are present mainly as monoterpenes (eugenol and geraniol) which have been reported to play a role in plant defense in leaves in other plant species as Picea (Faldt et al., 2003), pine (Himejima et al., 1992), or pistacia (Monaco et al., 1982) and in flavor in fruits as honeysuckle (Chmiel et al., 2013) or strawberry (Araguez et al., 2013). Also the terpene derived plant growth regulators abscisic acid and gibberellins are abundant, consistent with the physiological status of the plant in the flowering state.

An insight into phenolic compounds reveals that phenylpropanoids, flavonoids and anthocyanins biosynthetic genes are the most abundant. Considering the abundance of transcripts of the shikimate pathway, auxin biosynthesis and flavonol-anthocyanin pathway in leaves, data suggests that part of the metabolic flux is directed to feed auxin synthesis 
at the chorismic acid branching point, in addition to feeding flavonol-anthocyanin synthesis (Jorgensen et al., 2005).

In a previous work a ripening study was performed, showing changes in flavonol-anthocyanin pathway core genes expression, and bioactive accumulation in the three stages (green, red, black) of maturation in fruit (Garcia-Seco et al., 2015). In this work, fruit flavonoid, and anthocyanin biosynthesis was demonstrated. However, the high levels of catechins in green fruit without an increase in the expression of the corresponding biosynthetic genes suggested that catechins were probably originated in another part of the plant; this hypothesis was further supported in the other maturation stages in which, the accumulation of bioactives is not fully supported by the increase in gene expression. Finally, changes in gene expression along maturation were more evident in the red stage. Based on these facts, the present experiment was carried out to explore the possibility of flavonols translocation from leaves to growing fruits. The working hypothesis was set to demonstrate that leaves would actively synthetize flavonols and these would be translocated to supply anthocyanin synthesis in fruits, so leaf samples were taken in the same moment, when plants showed green, red and black fruits. To reinforce our hypothesis, leaf bioactives were also quantified at early flowering, when no fruits were present, resulting in lower levels of total phenolics and total flavonoids (data not shown). This data supports our hypothesis of increased leaf flavonol synthesis along the plant's cycle (Wang et al., 2009) in order to supply the fruit in its early stages.

Our results show that flavonol biosynthesis takes place in different organs, therefore they need to be accumulated and/or transported. An overexpression of chalcone synthase (RuCHS) coupled to $\mathrm{RuCHI} 2$ and $\mathrm{RuF} 3 \mathrm{H}$, is consistent with the higher concentration of flavonols found in leaves (Table 3) and supports the active synthesis therein. Interestingly, fruits and leaves showed expression of the MATE, ABC transporters, glutathione-S-transferases and glycosyltransferases (GSTs) involved in intracellular transport and glycosylation of anthocyanins, proanthocyanidins, and flavonols to vacuoles (Gomez et al., 2011; Zhao, 2015; Perez-Diaz et al., 2016). Therefore, flavonols and catechins are being actively glycosylated and consequently stored in each organ. However, the higher concentration of flavonols in leaves together with transporters (ABC and glutathione-S-transferases) overexpression and the RuGST1 and RuGST2 qPCR expression (Figure 8), indicates that they may as well be translocated to other tissues, consistent with the reported long distance transport demonstrated in A. thaliana by MATE and ABC transporters (Buer et al., 2007). Consequently, based in data from InterProScan Families Distribution overexpressed in leaves (Figure 4), the abundance of UDP-glycosiltransferases and the overexpression of $\mathrm{ABC}$ and glutathione-S-transferases transporters in leaves reinforce the hypothesis that flavonols synthetized in leaves are being translocated to fruits (Petrussa et al., 2013).

The overexpression of the isoform RuPAL1 over the RuPAL2 in fruits (Figure 8) is consistent with the complex role of this enzyme in directing the carbon flux to the flavonol pathway in key points of fruit ripening (Garcia-Seco et al., 2015; Dastmalchi et al., 2017) and further supports the initial activation of

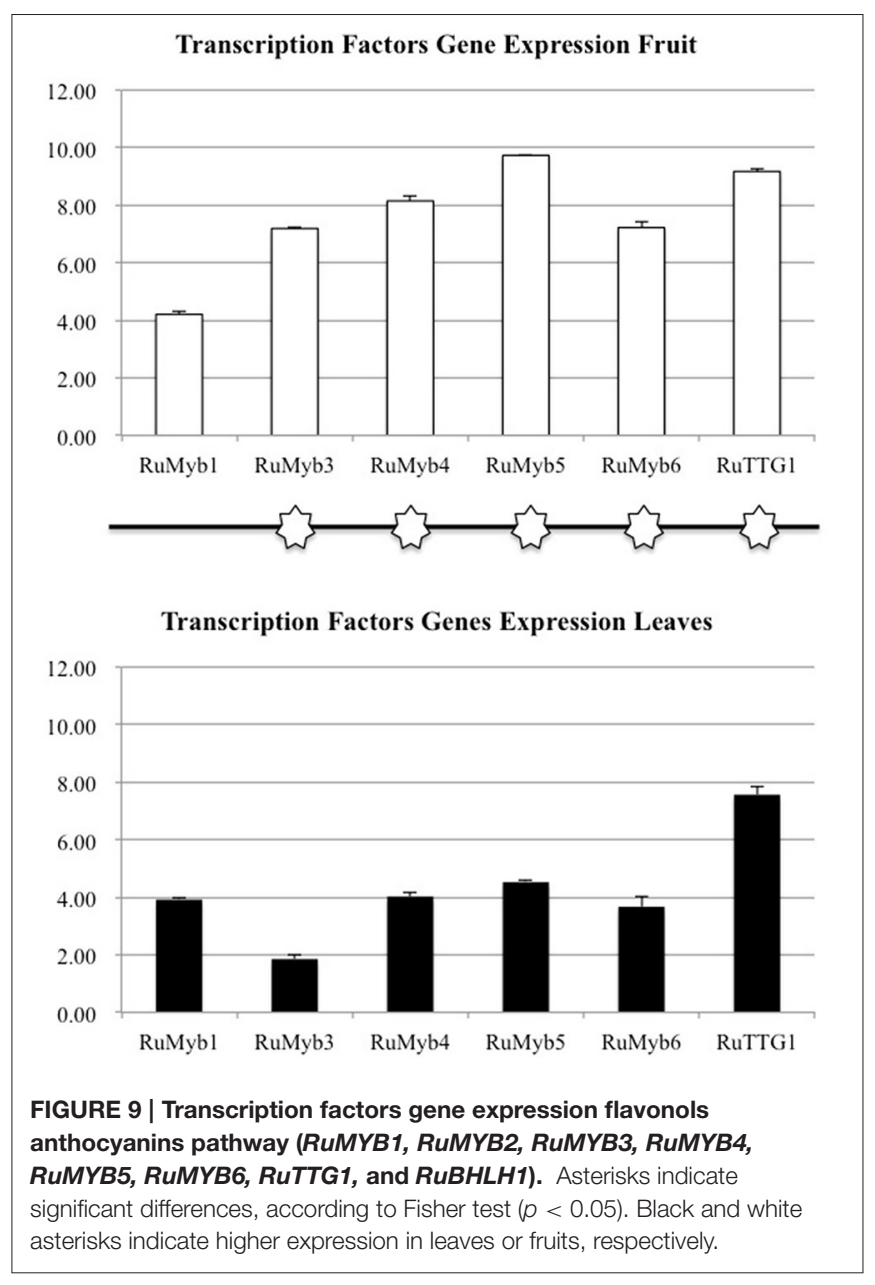

the flavonol synthesis in fruits, together with the presence of cholrogenic acid identified therein (Table 4). Interestingly, the almost two-fold expression of $R u D F R$ and $R u L A R$ in fruits suggests that accumulation of catechins (Table 3 ) is taking place in fruits, as these compounds are usually accumulated in seeds (Zhang et al., 2014) and is supported by the presence of catechins only in fruits (Tables 4, 5), probably synthetized from the quercetins originated in leaves. The downregulation of RuANS is consistent with the mid ripe status of blackberries, that still have not started to massively accumulate anthocyanins (Garcia-Seco et al., 2015).

Different branches of the flavonoid pathway are highly regulated at the transcriptional level, especially by MYB transcription factors (Czemmel et al., 2012). Transcription factors play an important role in flavonoid biosynthesis (Matsui et al., 2007) regulating some genes co-expression to stimulate or inhibit the accumulation of flavonoids. Based on a previous study on blackberry (Garcia-Seco et al., 2015), homologous regulatory genes (RuMYB1, RuMYB3, RuMYB4, RuMYB5, RuMYB6, RuTT2) were found and their expression was also evaluated (Figure 9).

The higher expression of the activating flavonol biosynthetic pathway transcription factor genes in fruit as compared 
TABLE 3 | Quantification of total phenolics, flavonols, and anthocyanins in blackberry fruits and leaves.

\begin{tabular}{|c|c|c|c|}
\hline Sample & $\begin{array}{l}\text { Total phenols } \mathrm{mg} \text { gallic acid } \\
\text { equivalents } / 100 \mathrm{~g} \text { fresh weigh }\end{array}$ & $\begin{array}{l}\text { Total flavonols } \mathrm{mg} \text { catechin acid } \\
\text { equivalents } / 100 \mathrm{~g} \text { fresh weigh }\end{array}$ & $\begin{array}{c}\text { Total anthocyanins } \mathrm{mg} \text { cyanidin-3-O-glucoside } \\
\text { acid equivalents } / 100 \mathrm{~g} \text { fresh weigh }\end{array}$ \\
\hline Leaves & ${ }^{*} 1876.56 \pm 32.47$ & ${ }^{*} 06.24 \pm 6.79$ & $28.40 \pm 0.78$ \\
\hline Fruit & $560.60 \pm 8.23$ & $34.70 \pm 1.41$ & ${ }^{*} 64.70 \pm 1.56$ \\
\hline
\end{tabular}

Data is the average of three replicates. Asterisks indicate significant differences, according to Fisher test $(p<0.05)$.

TABLE 4 | Mass spectral data for identification of phenolic acids, flavonols and anthocyanins in blackberry fruits (A) by LC/MS data.

\begin{tabular}{|c|c|c|c|c|c|c|c|c|}
\hline \multirow[t]{2}{*}{ Peak no. } & \multirow[t]{2}{*}{ Compound } & \multicolumn{4}{|c|}{ Positive ionization mode (+) } & \multicolumn{3}{|c|}{ Negative ionization mode (-) } \\
\hline & & Rt (min) & Selected ion & $\mathrm{m} / \mathrm{z}$ exp. & Fragment ions* & Selected ion & $m / z \exp$ & Fragment ions* \\
\hline 1 & chlorogenic acid (3-O-caffeoylquinic ac.) & $0.8-1.2$ & ND & - & - & {$[\mathrm{M}-\mathrm{H} \text {-caffeoyl }]^{-}$} & 190.6 & - \\
\hline 2 & cyanidin-3-O-glucoside & $2.1-4.4$ & {$[\mathrm{M}+\mathrm{H}]^{+}$} & 449.0 & 287.0 & {$[\mathrm{M}-\mathrm{H}]-$} & 446.9 & 284.7 \\
\hline 3 & $(+)$-catechin & 1.8. -4.1 & ND & - & - & {$[\mathrm{M}-\mathrm{H}]-$} & 288.8 & - \\
\hline 7 & (-)-epicatechin & $4.5-5.1$ & ND & - & - & {$[\mathrm{M}-\mathrm{H}]-$} & 288.8 & - \\
\hline
\end{tabular}

${ }^{\star}$ Peaks corresponding to the aglycon moiety.

ND- non detected.

TABLE 5 | Mass spectral data for identification of phenolic acids, flavonols and anthocyanins in blackberry leaves (B) by LC/MS data.

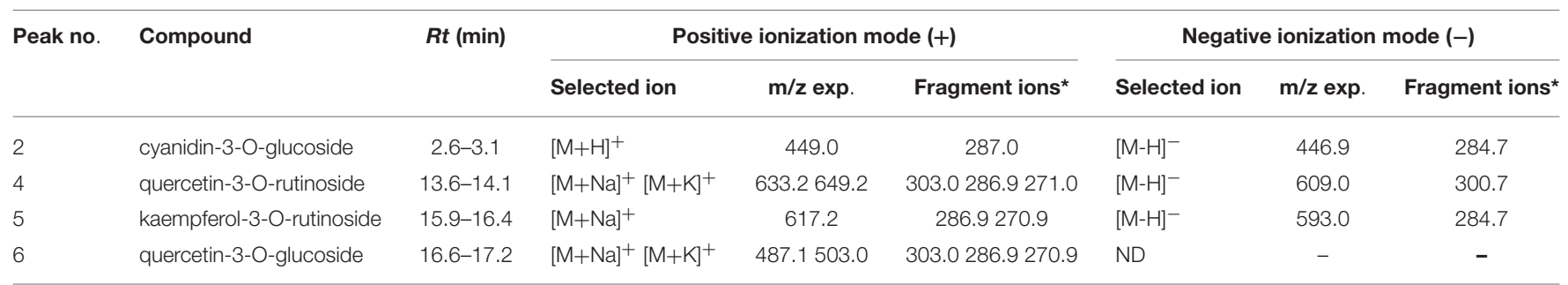

*Peaks corresponding to the aglycon moiety.

$N D$ - non detected.

to leaves (Figure 9; RuMYB1, RuMYB3, and RuMYB5) is consistent with the massive synthesis of anthocyanin precursors in fruits that is eventually going to take place during ripening. RuMYB1, RuMYB3, and RuMYB5 are positive regulators of $R u D F R, R u A N R$, and $R u L A R$, leading to increased catechin synthesis, precursors of proanthocyanidins, that are accumulating in fruits (Table 4). RuMYB1 encodes a homolog of FaMYB10, which plays a major role in the regulation of anthocyanin and phenylpropanoid metabolism during ripening of Fragaria $x$ ananassa fruits (Kadomura-Ishikawa et al., 2015). RuMYB5 is homologous to AtMYB5 in Arabidopsis, and the AtMYB5 protein interacts with TTG1 and TT2, two important transcription factors regulating the proanthocyanidin biosynthetic pathway (Baudry et al., 2004). The expression of the RuMYB5 in leaves is low, while it is quite high in fruits, consistent with the better activation of core pathway for proanthocyanidin synthesis in fruits (RuDFR, RuANR, $R u L A R$; Figure 8). Therefore, the enhanced expression of RuMYB1, 3, and 5 on fruits reflects an activation of the pathway leading to proanthocyanidins, data supported by the HPLC-MS analysis, where chlorogenic acid, cyanidin-3-Oglucoside and the catechols (+)-catechin and (-)-epicatechin, precursors of proanthocyanidins were identified in fruits (Tables 4, 5).

RuMYB6 encodes a homolog of GmMYB12B2 from Glycine $\max$, a transcription factor that activates $C H S$ in soybean and Arabidopsis. In fact, GmMYB12B2, induces expression of $P A L$, CHS, and FLS in Arabidopsis (Li et al., 2013). Our results show the activation of RuPAL and RuFLS expression in fruits. The higher expression of RuMYB6 in red fruit as compared to leaves, suggests that the massive synthesis of anthocyanins associated to maturation is going to start, as anticipated by the higher expression of RuMYB6 in fruits; until this happens, flavonoids are synthesized in leaves and then translocated to fruits.

RuMYB4 encodes a homolog of AtMYB4, an inhibitor of $\mathrm{C} 4 \mathrm{H}$ expression in Arabidopsis (Jin et al., 2000; Zhao et al., 2007). It shows high expression in red fruit consistent with the repression of $\mathrm{RuC4H}$ in fruits, while leaves show a lower expression of this $R u M Y B 4$, consistent with higher expression of $\mathrm{RuC} 4 \mathrm{H}$ therein, and further supports the hypothesis.

In summary, transcription factors expression in fruits is higher than in leaves suggesting that the onset of maturation 


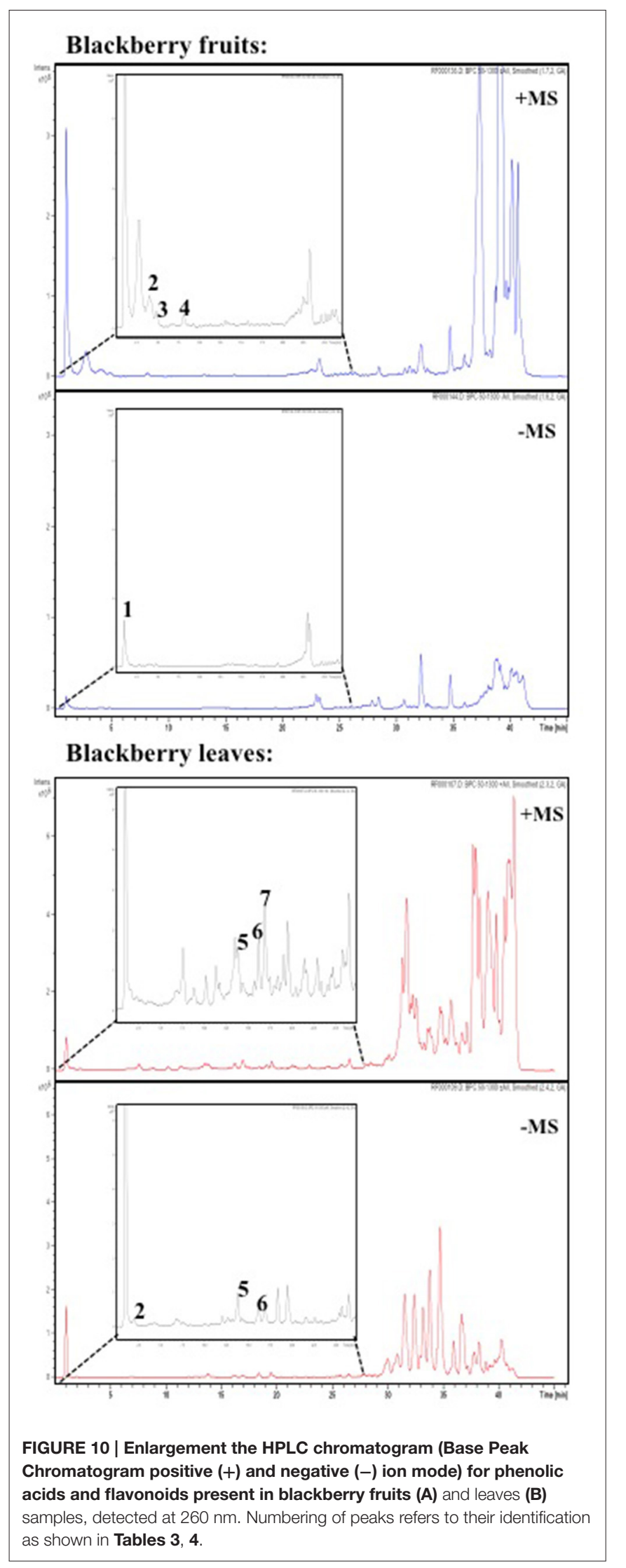

has been activated and will therefore need to receive an active precursor supply for anthocyanin synthesis and accumulation. The low expression of TF in leaves suggests that the pathway has already been activated, and therefore, TF have already played their role, so expression is lower; however, activity of core genes is reflected on flavonoid synthesis to fulfill fruit demand, and transport is supported by the overexpression of $\mathrm{ABC}$ and glutathione transferases genes in leaves and enhanced expression of RuGSTs in leaves. Finally, the six-fold concentration of flavonols in leaves as compared to fruits, and the accumulation of flavonols only in leaves as compared to catechins and cyanidin-3-glucoside, their downstream biosynthetic products in fruits, strongly support the translocation hypothesis to supply flavonol scaffoldings for first steps of fruit biosynthesis, which will be strongly activated when fruits achieve the mature state.

In conclusion, data of gene expression presented above is consistent with flavonol accumulation in red fruits that will be transformed into anthocyanins when fully mature (black; Garcia-Seco et al., 2015); conversely, in leaves the last part of the pathway (responsible of the production of flavonols and anthocyanins), is more expressed which makes sense, as these compounds also participate in plant defense. Moreover, these values are consistent with the expression levels of core and regulatory genes, supporting the working hypothesis of a strong synthesis of flavonols in leaves that are translocated to fruits to supply flavonol scaffoldings for first steps of fruit biosynthesis which synthesis will be strongly activated upon ripening; if this was so, flavonols will be translocated from leaves to fruits, creating a really efficient system for anthocyanin accumulation.

\section{AUTHOR CONTRIBUTIONS}

Conception and design of the work: FG and BR. Field work and sampling: JL, BR, EG. Analysis of the samples: AGV, AG, EG. Interpretation of the data: AGV, BR, JL, AG, EG. Drafting the work and revising: BR. Final approval of the version to be published: BR, FG.

\section{FUNDING}

Proyect funded By Ministerio de Economía y Competitividad: AGL-2013-45189-R. Grant reference: BES-2014-069990.

\section{ACKNOWLEDGMENTS}

Authors thank for Agricola El Bosque S.L. "La Canastita" for providing help in blackberry crop.

\section{SUPPLEMENTARY MATERIAL}

The Supplementary Material for this article can be found online at: http://journal.frontiersin.org/article/10.3389/fpls.2017. 00472/full\#supplementary-material 


\section{REFERENCES}

Adams, M. D., Kelley, J. M., Gocayne, J. D., Dubnick, M., Polymeropoulos, M. H., Xiao, H., et al. (1991). Complementary-DNA sequencing - expressed sequence tags and human genome project. Science 252, 1651-1656. doi: $10.1126 /$ science. 2047873

Agati, G., Azzarello, E., Pollastri, S., and Tattini, M. (2012). Flavonoids as antioxidants in plants: location and functional significance. Plant Sci. 196, 67-76. doi: 10.1016/j.plantsci.2012.07.014

Araguez, I., Hoffmann, T., Osorio, S., Rambla, J. L., Medina-Escobar, N., Granell, A., et al. (2013). Eugenol production in Achenes and receptacles of strawberry fruits is catalyzed by synthases exhibiting distinct kinetics. Plant Physiol. 163, 946-958. doi: 10.1104/pp.113.224352

Baudry, A., Heim, M. A., Dubreucq, B., Caboche, M., Weisshaar, B., and Lepiniec, L. (2004). TT2, TT8, and TTG1 synergistically specify the expression of BANYULS and proanthocyanidin biosynthesis in Arabidopsis thaliana. Plant J. 39, 366-380. doi: 10.1111/j.1365-313X.2004.02138.x

Brunetti, C., Di Ferdinando, M., Fini, A., Pollastri, S., and Tattini, M. (2013). Flavonoids as antioxidants and developmental regulators: relative significance in plants and humans. Int. J. Mol. Sci. 14, 3540-3555. doi: 10.3390/ijms14023540

Buer, C. S., Muday, G. K., and Djordjevic, M. A. (2007). Flavonoids are differentially taken up and transported long distances in Arabidopsis. Plant Physiol. 145, 478-490. doi: 10.1104/pp.107.101824

Cassidy, A., O’Reilly, E. J., Kay, C., Sampson, L., Franz, M., Forman, J. P., et al. (2011). Habitual intake of flavonoid subclasses and incident hypertension in adults. Am. J. Clin. Nutr. 93, 338-347. doi: 10.3945/ajcn.110.006783

Chmiel, T., Dymerski, T., Kupska, M., Wardencki, W., Gorecki, T., and Namiesnik, J. (2013). "Characterization of flavor profile and quantification of bioactive terpenes and macroelements in Polish blue honeysuckle berries (Lonicera caerulea L.) by GC x GC-TOFMS and cITP-cITP-CON," in Abstracts of Papers of the American Chemical Society (Poland), 245.

Conesa, A., and Gotz, S. (2008). Blast2GO: a comprehensive suite for functional analysis in plant genomics. Int. J. Plant Genomics. 2008:619832. doi: 10.1155/2008/619832

Czemmel, S., Heppel, S. C., and Bogs, J. (2012). R2R3 MYB transcription factors: key regulators of the flavonoid biosynthetic pathway in grapevine. Protoplasma 249 (Suppl. 2), S109-S118. doi: 10.1007/s00709-012-0380-z

Daayf, F., El Hadrami, A., El-Bebany, A. E., Henriquez, M. A., Yao, Z., Derksen, H., et al. (2012). Phenolic compounds in plant defense and pathogen counter-defense mechanisms. Recent Adv. Polyphenol Res. 3, 191-208. doi: 10.1002/9781118299753.ch8

Dastmalchi, M., Chapman, P., Yu, J. S., and Austin, R., Dhaubhadel, S. (2017). Transcriptomic evidence for the control of soybean root isoflavonoid content by regulation of overlapping phenylpropanoid pathways. BMC Genomics 18:70. doi: 10.1186/s12864-016-3463-y

Dong, C., Hu, H. G., Hu, Y. L., and Xie, J. H. (2016). Metabolism of flavonoids in novel banana germplasm during fruit development. Front. Plant Sci. 7:1291. doi: 10.3389/fpls.2016.01291

Falcone Ferreyra, M. L., Rius, S. P., and Casati, P. (2012). Flavonoids: biosynthesis, biological functions, and biotechnological applications. Front. Plant Sci. 3:222. doi: 10.3389/fpls.2012.00222

Faldt, J., Martin, D., Miller, B., Rawat, S., and Bohlmann, J. (2003). Traumatic resin defense in Norway spruce (Picea abies): methyl jasmonate-induced terpene synthase gene expression, and cDNA cloning and functional characterization of (+)-3-carene synthase. Plant Mol. Biol. 51, 119-133. doi: 10.1023/A:1020714403780

Feresin, R. G., Huang, J., Klarich, D. S., Zhao, Y., Pourafshar, S., Arjmandi, B. H., et al. (2016). Blackberry, raspberry and black raspberry polyphenol extracts attenuate angiotensin II-induced senescence in vascular smooth muscle cells. Food Funct. 7, 4175-4187. doi: 10.1039/C6FO00743K

Fuchs, D., Nyakayiru, J., Draijer, R., Mulder, T. P., Hopman, M. T., Eijsvogels, T. M., et al. (2016). Impact of flavonoid-rich black tea and beetroot juice on postprandial peripheral vascular resistance and glucose homeostasis in obese, insulin-resistant men: a randomized controlled trial. Nutr. Metab. (Lond). 13, 10. doi: 10.1186/s12986-016-0094-x

Garcia-Seco, D., Bonilla, A., Algar, E., Garcia-Villaraco, A., Manero, J. G., and Ramos-Solano, B. (2013). Enhanced blackberry production using Pseudomonas fluorescens as elicitor. Agron. Sus. Dev. 33, 385-392. doi: 10.1007/s13593-012-0103-Z

Garcia-Seco, D., Zhang, Y., Gutierrez-Manero, F. J., Martin, C., and Ramos-Solano, B. (2015). Application of Pseudomonas fluorescens to blackberry under field conditions improves fruit quality by modifying flavonoid metabolism. PLoS ONE 10:23. doi: 10.1371/journal.pone.0142639

Gomez, C., Conejero, G., Torregrosa, L., Cheynier, V., Terrier, N., and Ageorges, A. (2011). In vivo grapevine anthocyanin transport involves vesicle-mediated trafficking and the contribution of anthoMATE transporters and GST. Plant J. 67, 960-970. doi: 10.1111/j.1365-313X.2011.04648.x

Giusti, M. M., and Wrolstad, R. E. (2001). Characterization and measurement of anthocyanins by UV-visible spectroscopy. Curr. Protoc. Food Anal. Chem. F1.2.1-F1.2.13. doi: 10.1002/0471142913.faf0102s00

Gutiérrez-Mañero, F., García-Villaraco, A., Lucas, J., Gutiérrez, E., and Ramos-Solano, B. (2015). Inoculant/elicitation technology to improve bioactive/phytoalexin contents in functional foods. Int. J. Curr. Microbiol. Appl. Sci. 4, 224-241.

Himejima, M., Hobson, K. R., Otsuka, T., Wood, D. L., and Kubo, I. (1992). Antimicrobial terpenes from oleoresin of ponderosa pine tree pinus-ponderosa - a defense-mechanism against microbial invasion. J. Chem. Ecol. 18, 1809-1818. doi: 10.1007/BF02751105

Jia, Z., Tang, M. C., and Wu, J. M. (1999). The determination of flavonoid contents in mulberry and their scavenging effects on superoxide radicals. Food Chem. 64, 555-559. doi: 10.1016/S0308-8146(98)00102-2

Jin, H. L., Cominelli, E., Bailey, P., Parr, A., Mehrtens, F., Jones, J., et al. (2000). Transcriptional repression by AtMYB4 controls production of UV-protecting sunscreens in Arabidopsis. Embo J. 19, 6150-6161. doi: 10.1093/emboj/19.22.6150

Jorgensen, K., Rasmussen, A. V., Morant, M., Nielsen, A. H., Bjarnholt, N., Zagrobelny, M., et al. (2005). Metabolon formation and metabolic channeling in the biosynthesis of plant natural products. Curr. Opin. Plant Biol. 8, 280-291. doi: 10.1016/j.pbi.2005.03.014

Kadomura-Ishikawa, Y., Miyawaki, K., Takahashi, A., Masuda, T., and Noji, S. (2015). Light and abscisic acid independently regulated FaMYB10 in Fragaria $\mathrm{X}$ ananassa fruit. Planta 241, 953-965. doi: 10.1007/s00425-014-2228-6

Kim, H. G., Kim, G. S., Lee, J. H., Park, S., Jeong, W. Y., Kim, Y. H., et al. (2011). Determination of the change of flavonoid components as the defence materials of Citrus unshiu Marc. fruit peel against Penicillium digitatum by liquid chromatography coupled with tandem mass spectrometry. Food Chem. 128, 49-54. doi: 10.1016/j.foodchem.2011.02.075

Kim, J.-A. (2008). Mechanisms underlying beneficial health effects of tea catechins to improve insulin resistance and endothelial dysfunction. Endocr. Metab. Immune Disord. Drug Targets 8, 82-88. doi: 10.2174/187153008784534349

Koskimaki, J. J., Hokkanen, J., Jaakola, L., Suorsa, M., Tolonen, A., Mattila, S., et al. (2009). Flavonoid biosynthesis and degradation play a role in early defence responses of bilberry (Vaccinium myrtillus) against biotic stress. Eur. J. Plant Pathol. 125, 29-640. doi: 10.1007/s10658-009-9511-6

Li, X. W., Li, J. W., Zhai, Y., Zhao, Y., Zhao, X., Zhang, H. J., et al. (2013). A R2R3-MYB transcription factor, GmMYB12B2, affects the expression levels of flavonoid biosynthesis genes encoding key enzymes in transgenic Arabidopsis plants. Gene 532, 72-79. doi: 10.1016/j.gene.2013.09.015

Lotito, S. B., and Frei, B. (2004). The increase in human plasma antioxidant capacity after apple consumption is due to the metabolic effect of fructose on urate, not apple-derived antioxidant flavonoids. Free Radic. Biol. Med. 37, 251-258. doi: 10.1016/j.freeradbiomed.2004.04.019

Makoi, J., Belane, A. K., Chimphango, S. B. M., and Dakora, F. D. (2010). Seed flavonoids and anthocyanins as markers of enhanced plant defence in nodulated cowpea (Vigna unguiculata L. Walp.). Field Crops Res. 118, 21-27. doi: 10.1016/j.fcr.2010.03.012

Martin, C., Butelli, E., Petroni, K., and Tonelli, C. (2011). How can research on plants contribute to promoting human health? Plant Cell 23, 1685-1699. doi: 10.1105/tpc.111.083279

Matsui, K., Umemura, Y., and Ohme-Takagi, M. (2007). AtMYBL2, a protein with a single MYB domain, acts as a negative regulator of anthocyanin biosynthesis in Arabidopsis. Plant Cell Physiol. 55, 954-967. doi: 10.1111/j.1365-313x.2008. 03565.x

Monaco, P., Previtera, L., and Mangoni, L. (1982). Terpenes in PISTACIAplants - a possible defense role for monoterpenes against gall-forming 
aphids. Phytochemistry 21, 2408-2410. doi: 10.1016/0031-9422(82) 85219-9

Nakabayashi, R., Yonekura-Sakakibara, K., Urano, K., Suzuki, M., Yamada, Y., Nishizawa, T., et al. (2014). Enhancement of oxidative and drought tolerance in Arabidopsis by overaccumulation of antioxidant flavonoids. Plant J. 77, 367-379. doi: 10.1111/tpj.12388

Paredes-López, O., Cervantes-Ceja, M. L., Vigna-Pérez, M., and Hernández-Pérez, T. (2010). Berries: improving human health and healthy aging, and promoting quality life-a review. Plant Foods Hum. Nutr. 65, 299-308. doi: 10.1007/s11130010-0177-1

Perez-Diaz, R., Madrid-Espinoza, J., Salinas-Cornejo, J., Gonzalez-Villanueva, E., and Ruiz-Lara, S. (2016). Differential roles for VviGST1, VviGST3, and VviGST4 in proanthocyanidin and anthocyanin transport in Vitis vinifera. Front. Plant Sci. 7:1166. doi: 10.3389/fpls.2016.01166

Petrussa, E., Braidot, E., Zancani, M., Peresson, C., Bertolini, A., Patui, S., et al. (2013). Plant flavonoids-biosynthesis, transport and involvement in stress responses. Int. J. Mol. Sci. 14, 14950-14973. doi: 10.3390/ijms140714950

Ramakrishna, A., and Ravishankar, G. A. (2011). Influence of abiotic stress signals on secondary metabolites in plants. Plant Signal. Behav. 6, 1720-1731. doi: $10.4161 /$ psb.6.11.17613

Ramos-Solano, B., Garcia-Villaraco, A., Gutierrez-Manero, F. J., Lucas, J. A., Bonilla, A., and Garcia-Seco, D. (2014). Annual changes in bioactive contents and production in field-grown blackberry after inoculation with Pseudomonas fluorescens. Plant Physiol. Biochem. 74, 1-8. doi: 10.1016/j.plaphy.2013.10.029

Sarkar, D., Orwat, J., Hurburt, T., Woods, F., Pitts, J. A., and Shetty, K. (2016). Evaluation of phenolic bioactive-linked functionality of blackberry cultivars targeting dietary management of early stages type-2 diabetes using in vitro models. Sci. Hortic. 212, 193-202. doi: 10.1016/j.scienta.2016.10.003

Tavares, L., Figueira, I., McDougall, G. J., Vieira, H. L., Stewart, D., Alves, P. M., et al. (2013). Neuroprotective effects of digested polyphenols from wild blackberry species. Eur. J. Nutr. 52, 225-236. doi: 10.1007/s00394-012-0307-7

Vance, C. P., Miller, S. S., Gregerson, R. G., Samac, D. A., Robinson, D. L., and Gantt, J. S. (1995). Alfalfa NADH-dependent glutamate synthase - structure of the gene and importance in symbiotic N-2 fixation. Plant J. 8, 345-358. doi: 10.1046/j.1365-313X.1995.08030345.x

Wang, S. Y., and Lin, H. S. (2000). Antioxidant activity in fruits and leaves of blackberry, raspberry, and strawberry varies with cultivar and developmental stage. J. Agric. Food Chem. 48, 140-146. doi: 10.1021/jf9908345
Wang, X., Wang, X. W., Wang, L. K., Feng, Z. X., and Zhang, X. G. (2010). A review on the processing and analysis of next-generation RNA-seq data. Progr. Biochem. Biophys. 37, 834-846. doi: 10.3724/SP.J.1206.2009.00151

Wang, Z., Gerstein, M., and Snyder, M. (2009). RNA-Seq: a revolutionary tool for transcriptomics. Nat. Rev. Genet. 10, 57-63. doi: 10.1038/nrg2484

Went, F. (1933). The significance of substances (Auxin) for growth, photo- and geotropic curvatures. Naturwissenschaften 21, 1-7. doi: 10.1007/BF01505696

$\mathrm{Xu}, \mathrm{B}$. J., and Chang, S. K. C. (2007). A comparative study on phenolic profiles and antioxidant activities of legumes as affected by extraction solvents. J. Food Sci. 72, S159-S166. doi: 10.1111/j.1750-3841.2006.00260.x

Yauk, Y.-K., Ged, C., Wang, M. Y., Matich, A. J., Tessarotto, L., Cooney, J. M., et al. (2014). Manipulation of flavour and aroma compound sequestration and release using a glycosyltransferase with specificity for terpene alcohols. Plant J. 80, 317-330. doi: 10.1111/tpj.12634

Zhang, S. T., Cui, Y., and Sun, B. S. editors., (2014). "Preparative isolation of monomer catechins and oligomer procyanidin fractions from grape seed extracts by high-speed counter-current chromatography," in 37th World Congress of Vine and Wine/12th General Assembly of the OIV Nov 09-14 (Mendoza: CEDEX A: E D P Sciences 2014).

Zhao, J. (2015). Flavonoid transport mechanisms: how to go, and with whom. Trends Plant Sci. 20, 576-585. doi: 10.1016/j.tplants.2015.06.007

Zhao, J. F., Zhang, W. H., Zhao, Y., Gong, X. M., Guo, L., Zhu, G. L., et al. (2007). SAD2, an importin beta-like protein, is required for UV-B response in Arabidopsis by mediating MYB4 nuclear trafficking. Plant Cell 19, 3805-3818. doi: $10.1105 /$ tpc. 106.048900

Conflict of Interest Statement: The authors declare that the research was conducted in the absence of any commercial or financial relationships that could be construed as a potential conflict of interest.

Copyright (C) 2017 Gutierrez, García-Villaraco, Lucas, Gradillas, GutierrezMañero and Ramos-Solano. This is an open-access article distributed under the terms of the Creative Commons Attribution License (CC BY). The use, distribution or reproduction in other forums is permitted, provided the original author(s) or licensor are credited and that the original publication in this journal is cited, in accordance with accepted academic practice. No use, distribution or reproduction is permitted which does not comply with these terms. 